\title{
A Readily-Reconfigurable Continuous-Stirred Tank Photochemical Reactor Platform
}

Daniel Francis, A. John Blacker, * Nikil Kapur, * and Stephen P. Marsden*

\section{Supporting Information}

$\begin{array}{ll}\text { Page Contents } \\ 2 \quad \text { General experimental } \\ 4 \quad \text { Hydroamination reaction optimisation table } \\ 5 \quad \text { Experimental and compound characterisation } \\ 12 \quad{ }^{1} \mathrm{H} \text { and }{ }^{13} \mathrm{C} \text { spectra for } \mathbf{2 a - d}, \mathbf{3}, \mathbf{6 a - f} \text {, benzophenone } \\ 24 & \text { Crude }{ }^{1} \mathrm{H} \text { NMR spectra of } \mathbf{2 a - d} \text { (conversion/selectivity measurements) } \\ 28 & \text { References }\end{array}$




\section{General experimental}

The CSTRs used are the fReactor platform, with the Photo Flow module. Both are used as available from asynt.com (https://www.asynt.com/product/freactor-classic-complete-kit/ and https://www.asynt.com/product/freactor-photo-flow/). The individual units have an internal volume of $1.77 \mathrm{~mL}$ without the stirrer bar and $1.63 \mathrm{~mL}$ with. Residence times are therefore calculated based upon a volume per reactor of $1.63 \mathrm{~mL}$ - the volume within linking tubing is disregarded since these are 'dark' regions with no illumination. Steady state is defined as the point where $>2$ reactor volumes has passed through the system. LEDs used are either $365 \mathrm{~nm}$ (nominal radiant light output, $4.3 \mathrm{~W}$ ) or $460 \mathrm{~nm}(3.9 \mathrm{~W})$. For the biphasic gas/liquid reactions, the effective liquid reactor volume is estimated to be half that of the normal reactor as in Figure S1 below, and hence residence times are adjusted accordingly:

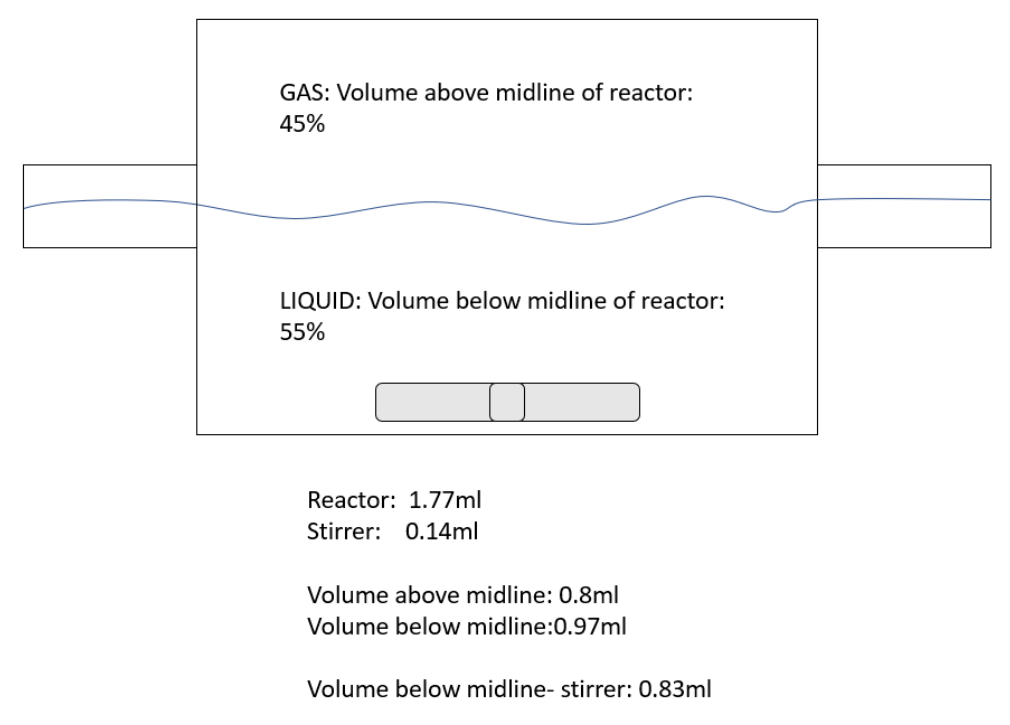

Figure S1: schematic of behaviour of CSTRs under biphasic gas/liquid conditions

Commercially available starting materials were obtained from Sigma-Aldrich, Fluorochem,

Acros and Alfa Aesar. All non-aqueous reactions were performed under nitrogen

atmosphere unless otherwise stated. Water-sensitive reactions were performed in

anhydrous solvents in oven-dried glassware cooled under nitrogen before use. Anhydrous

dichloromethane (DCM), anhydrous toluene were obtained from a PureSolv MD5 Purification

System. All other solvents used were of chromatography or analytical grade.

Thin layer chromatography (TLC) was performed using aluminium backed silica (Merck silica 
gel 60 F254) plates obtained from Merck. An ultraviolet lamp $(\lambda \max =254 \mathrm{~nm})$ and $\mathrm{KMnO}_{4}$ stain were used for visualization. Flash column chromatography was performed using silica gel 60 (35-70 $\mu \mathrm{m}$ particles) supplied by Merck. A Bruker Daltonics micrOTOF spectrometer with electrospray (ES) ionisation source was used for high-resolution mass spectrometry (HRMS). Proton $\left({ }^{1} \mathrm{H}\right)$ and carbon $\left({ }^{13} \mathrm{C}\right)$ NMR data was collected on a Bruker 300,400 or 500 $\mathrm{MHz}$ spectrometer using $\mathrm{CDCl}_{3}$ or d4-methanol as solvents. Data was collected at $300 \mathrm{~K}$ unless otherwise stated. Chemical shifts $(\delta)$ are given in parts per million (ppm) and they are referenced to the residual solvent peak. Coupling constants $(J)$ are reported in $\mathrm{Hertz}(\mathrm{Hz})$ and splitting patterns are reported in an abbreviated manner: app. (apparent), s (singlet), d (doublet), $\mathrm{t}$ (triplet), q (quartet), m (multiplet), br. (broad). Assignments were made using COSY, DEPT, HMQC and NOESY experiments. 


\section{Hydroamination reaction optimisation}

Reactions were set up as for general method C, with deviations from the general method listed in Table S1 below. Conversion was measured by consumption of starting enecarbamate and isolated yields were recorded after purification of an aliquot at steady state.
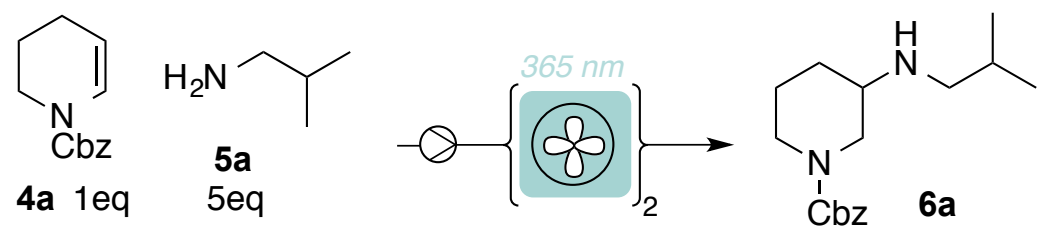

$\left[\operatorname{Ir}\left(\mathrm{dF}\left(\mathrm{CF}_{3}\right) \mathrm{ppy}\right)_{2}(\mathrm{dtbbpy})\right] \mathrm{PF}_{6}(0.5 \mathrm{~mol} \%)$

TRIP thiol (50 mol\%)

toluene

Table S1: optimisation of continuous photoredox hydroaminations

\begin{tabular}{|c|c|c|c|c|c|}
\hline$\#$ & $\begin{array}{c}\text { Flow rate } \\
\left(\mathrm{t}_{\text {res }}\right)\end{array}$ & {$[\mathrm{C}]$} & Other & $\begin{array}{c}\text { Conversion }^{\mathrm{a}} \\
\text { (Isolated yield) }\end{array}$ & STY (g/d/R)* \\
\hline 1 & $50 \mu \mathrm{Lmin}^{-1}(32.6 \mathrm{~min})$ & $50 \mathrm{mM}$ & $\begin{array}{c}1 \text { Freactor unit } \\
\text { only }\end{array}$ & $38 \%$ & $0.39^{\mathrm{b}}$ \\
\hline 2 & $50 \mu \mathrm{Lmin}^{-1}(65.2 \mathrm{~min})$ & $50 \mathrm{mM}$ & - & $56 \%$ & $0.29^{\mathrm{b}}$ \\
\hline 3 & $50 \mu \mathrm{Lmin}^{-1}(65.2 \mathrm{~min})$ & $50 \mathrm{mM}$ & Cat B $(0.5 \mathrm{~mol} \%)^{\mathrm{c}}$ & $30 \%$ & $0.16^{\mathrm{b}}$ \\
\hline 4 & $50 \mu \mathrm{Lmin}^{-1}(65.2 \mathrm{~min})$ & $100 \mathrm{mM}$ & - & $48 \%(40 \%)$ & $0.42^{\mathrm{c}}$ \\
\hline 5 & $50 \mu \mathrm{Lmin}^{-1}(65.2 \mathrm{~min})$ & $50 \mathrm{mM}$ & $80^{\circ} \mathrm{C}$ & $51 \%$ & $0.27^{\mathrm{b}}$ \\
\hline 6 & $50 \mu \mathrm{Lmin}^{-1}(65.2 \mathrm{~min})$ & $100 \mathrm{mM}$ & $4: 1 \mathrm{Toluene}: \mathrm{THF}$ & $50 \%(45 \%)$ & $0.47^{\mathrm{c}}$ \\
\hline 7 & $50 \mu \mathrm{Lmin}^{-1}(65.2 \mathrm{~min})$ & $100 \mathrm{mM}$ & $100 \%$ TRIP thiol & $22 \%$ & $0.23^{\mathrm{b}}$ \\
\hline 8 & $50 \mu \mathrm{Lmin}^{-1}(65.2 \mathrm{~min})$ & $100 \mathrm{mM}$ & $20 \%$ TRIP thiol & $41 \%$ & $0.43^{\mathrm{b}}$ \\
\hline 9 & $50 \mu \mathrm{Lmin}^{-1}(65.2 \mathrm{~min})$ & $200 \mathrm{mM}$ & $4: 1$ Tol THF & $17 \%$ & $0.36^{\mathrm{b}}$ \\
\hline 10 & $50 \mu \mathrm{Lmin}^{-1}(65.2 \mathrm{~min})$ & $200 \mathrm{mM}$ & $4: 1$ Tol DCM & $42 \%(37 \%)$ & $0.78^{\mathrm{c}}$ \\
\hline
\end{tabular}

* STY- space/time yield based on grams per day per fReactor module; ${ }^{a}$ conversion measured by comparing product to unreacted enecarbamate in crude ${ }^{1} \mathrm{H} N \mathrm{NMR}$; amount based on ${ }^{\mathrm{b}}$ conversions or ${ }^{c}$ isolated yields. ${ }^{c}$ Cat $B \operatorname{Ir}[d F F p p y] 2-\left(4,4^{\prime}-d_{C F} b p y\right) P F_{6}$ 


\section{Experimental and compound characterisation}

1-(4-(Bromomethyl)phenyl)ethan-1-one 2a<smiles>CC(=O)c1ccc(CBr)cc1</smiles>

Homogeneous: 2a was synthesised using the general method using 4-methylacetophenone (500 $\mathrm{mM}$ final concentration) and $\mathrm{N}$-bromosuccinimide (525 $\mathrm{mM}$ final concentration) in anhydrous acetonitrile. The solution was injected into two sequential reactors at a flow rate of $1.5 \mathrm{mLmin}^{-1}\left(\mathrm{t}_{\text {res }}\right.$ 2.2mins). A $2 \mathrm{~mL}$ aliquot was taken for analysis which after column chromatography (5\% ethyl acetate in hexane) gave $\mathbf{2 a}$ isolated as a colourless oil (163 mg, 77\%; 90\% pure - contains 10\% 1,1dibromide).

Slurry: 2a was synthesised using the general method using 4-methylacetophenone (2 M final concentration) and $\mathrm{N}$-bromosuccinimide (total soluble/insoluble equivalent to $2.1 \mathrm{M}$ final concentration) in anhydrous acetonitrile. The slurry was injected into two sequential reactors at a flow rate of $1.25 \mathrm{mLmin}^{-1}$ ( $t_{\text {res }} 2.6$ mins) with the suspension constantly stirred in the syringe barrel. $A$ $2 \mathrm{~mL}$ aliquot was taken for analysis which after column chromatography (5\% ethyl acetate in hexane) gave $2 \mathrm{a}$ isolated as a white solid (538 $\mathrm{mg}, 65 \%$; $92 \%$ pure - contains $8 \%$ 1,1-dibromide).

Spectral data matches that reported. ${ }^{1}{ }^{1} \mathrm{H} N M R\left(501 \mathrm{MHz}, \mathrm{CDCl}_{3}\right) \delta_{\mathrm{H}} 7.91(2 \mathrm{H}, \mathrm{d}, J$ 8.4, H2), $7.46(2 \mathrm{H}$, d, J 8.4, H3), $4.48\left(2 \mathrm{H}, \mathrm{s}, \mathrm{CH}_{2} \mathrm{Br}\right), 2.58(3 \mathrm{H}, \mathrm{s}, \mathrm{Ac}) ;{ }^{13} \mathrm{C} \mathrm{NMR}\left(126 \mathrm{MHz}, \mathrm{CDCl}_{3}\right) \delta 197.4(\mathrm{C}=0), 142.9$ ( $\mathrm{ArC}), 136.9(\mathrm{ArC}), 129.3(\mathrm{ArCH}), 128.9(\mathrm{ArCH}), 32.2\left(\mathrm{CH}_{2} \mathrm{Br}\right), 26.7(\mathrm{Me})$.

1-(Bromomethyl)-4-(tert-butyl)benzene $\mathbf{2 b}$<smiles>CC(C)(C)c1ccc(CBr)cc1</smiles>

Homogeneous: $\mathbf{2 b}$ was synthesised using the general method using 4-tert-butyltoluene (500 mM final concentration) and $\mathrm{N}$-bromosuccinimide (525 mM final concentration) in anhydrous acetonitrile. The solution was injected into two sequential reactors at a flow rate of $4 \mathrm{mLmin}^{-1}\left(t_{\text {res }}\right.$ 0.815 mins). A $2.4 \mathrm{~mL}$ aliquot was taken for analysis, which after column chromatography $(2.5 \%$ ethyl acetate in hexane) gave $\mathbf{2 b}$ isolated as a white solid (201 mg, 74\%; 92\% pure - contains 8\% 1,1dibromide). 
Slurry: (2b) was synthesised using the general method using 4-tert-butyltoluene (2 M final concentration) and $\mathrm{N}$-bromosuccinimide (total soluble/insoluble equivalent to $2.1 \mathrm{M}$ final concentration) in anhydrous acetonitrile. The slurry was injected into two sequential reactors at a flow rate of $4 \mathrm{~mL} \mathrm{~min}^{-1}$ ( $t_{\text {res }} 0.815 \mathrm{mins}$ ) with the suspension constantly stirred in the syringe barrel. $A$ $1.2 \mathrm{~mL}$ aliquot was taken for analysis which after column chromatography (2.5\% ethyl acetate in hexane) gave $\mathbf{2 b}$ isolated as a white solid (314 $\mathrm{mg}$, 58\%; $95 \%$ pure - contains 5\% 1,1-dibromide). Spectral data matches that reported. ${ }^{11} \mathrm{H}$ NMR $\left(501 \mathrm{MHz}, \mathrm{CDCl}_{3}\right) \delta_{\mathrm{H}} 7.42-7.35(2 \mathrm{H}, \mathrm{m}, \mathrm{H2}), 7.36-$ $7.32(2 \mathrm{H}, \mathrm{m}, \mathrm{H} 3), 4.51\left(2 \mathrm{H}, \mathrm{s}, \mathrm{BrCH}_{2}\right), 1.35-1.31(9 \mathrm{H}, \mathrm{m}, \mathrm{tBu}) ;{ }^{13} \mathrm{C} \mathrm{NMR}\left(126 \mathrm{MHz}, \mathrm{CDCl}_{3}\right) \delta 151.7$ ( $\operatorname{ArC}), 134.9(\mathrm{ArC}), 128.9(\mathrm{ArCH}), 125.9(\mathrm{ArCH}), 34.8\left(\mathrm{C}^{\mathrm{t}} \mathrm{Bu}\right), 33.8\left(\mathrm{CH}_{2} \mathrm{Br}\right), 31.4(\mathrm{Me})$.

1-(Bromomethyl)-4-methoxybenzene 2c<smiles>COc1ccc(CBr)cc1</smiles>

Homogeneous: $2 \mathrm{c}$ was synthesised using the general method using 4-methylanisole (500 mM final concentration), $\mathrm{N}$-bromosuccinimide (525 mM final concentration in anhydrous 1,4-dioxane. The solution was injected into two sequential reactors at a flow rate of $4 \mathrm{mLmin}^{-1}$ ( $\mathrm{t}_{\text {res }} 0.815 \mathrm{mins}$ ). A 3 $\mathrm{mL}$ aliquot was taken for analysis which after column chromatography (5\% ethyl acetate in hexane) gave $\mathbf{2 c}$ isolated as an oil (175 mg, 58\%; 93\% pure - contains 7\% $p$-anisaldehyde).

Slurry: $2 \mathrm{c}$ was synthesised using the general method using 4-methylanisole ( $2 \mathrm{M}$ final concentration), $\mathrm{N}$-bromosuccinimide (total soluble/insoluble equivalent to $2.1 \mathrm{M}$ final concentration) in anhydrous 1,4-dioxane. The slurry was injected into two sequential reactors at a flow rate of $2 \mathrm{mLmin}^{-1}$ ( $\mathrm{t}_{\text {res }} 1.63$ $\mathrm{min}$ ) with the suspension constantly stirred in the syringe barrel. A $2 \mathrm{~mL}$ aliquot was taken for analysis which after column chromatography ( $5 \%$ ethyl acetate in hexane) gave $\mathbf{2 c}$ isolated as an isolated as an oil (568 mg, 71\%; 91\% pure - contains $9 \%$-anisaldehyde).

Spectral data matches that reported. ${ }^{2}{ }^{1} \mathrm{H}$ NMR $\left(501 \mathrm{MHz}, \mathrm{CDCl}_{3}\right) \delta_{\mathrm{H}} 7.33(2 \mathrm{H}, \mathrm{d}, J 8.8, \mathrm{H} 3), 6.87(2 \mathrm{H}$, d, J 8.8, H2), $4.51\left(2 \mathrm{H}, \mathrm{s}, \mathrm{CH}_{2} \mathrm{Br}\right), 3.81(3 \mathrm{H}, \mathrm{s}, \mathrm{OMe}) ;{ }^{13} \mathrm{C} \mathrm{NMR}\left(126 \mathrm{MHz}, \mathrm{CDCl}_{3}\right) \delta 159.8(\mathrm{ArC}), 130.6$ ( $\mathrm{ArCH}), 130.1(\mathrm{ArC}), 114.4(\mathrm{ArCH}), 55.5(\mathrm{OMe}), 34.1\left(\mathrm{CH}_{2} \mathrm{Br}\right)$. 
4'-(Bromomethyl)-[1,1'-biphenyl]-2-carbonitrile 2d<smiles>N#Cc1ccccc1-c1ccc(CBr)cc1</smiles>

Homogeneous: $\mathbf{2 d}$ was synthesised using the general method using 4'-methyl-[1,1'-biphenyl]-2carbonitrile ( $500 \mathrm{mM}$ final concentration) and $\mathrm{N}$-bromosuccinimide ( $525 \mathrm{mM}$ final concentration) in anhydrous acetonitrile. The solution was injected into two sequential reactors at a flow rate of 1.5 $\mathrm{mL} \mathrm{min}^{-1}$ ( $\mathrm{t}_{\text {res }} 2.17 \mathrm{mins}$ ). A $2 \mathrm{~mL}$ aliquot was taken for analysis which after column chromatography ( $5 \%$ ethyl acetate in hexane) gave $\mathbf{2} \mathbf{d}$ isolated as a white solid (164 $\mathrm{mg}, 61 \%$; $94 \%$ pure - contains $6 \%$ 1,1-dibromide).

Slurry: $\mathbf{2 d}$ was synthesised using the general method using 4'-methyl-[1,1'-biphenyl]-2-carbonitrile (1.5 $\mathrm{M}$ final concentration) and $\mathrm{N}$-bromosuccinimide (total soluble/insoluble equivalent to $1.575 \mathrm{M}$ final concentration in anhydrous 1,4 dioxane. The slurry was injected into two sequential reactors at a flow rate of $1.5 \mathrm{mLmin}^{-1}$ ( $\mathrm{t}_{\text {res }} 2.17 \mathrm{mins}$ ) with the suspension constantly stirred in the syringe barrel. A $2 \mathrm{~mL}$ aliquot was taken for analysis which after column chromatography (5\% ethyl acetate in hexane) gave $\mathbf{2 d}$ isolated as a white solid (340 $\mathrm{mg}$, 41\%; $96 \%$ pure - contains $4 \%$ 1,1-dibromide).

Spectral data matches that reported. ${ }^{11} \mathrm{H} N M R\left(501 \mathrm{MHz}, \mathrm{CDCl}_{3}\right) \delta_{\mathrm{H}} 7.77(1 \mathrm{H}, \mathrm{ddd}, \mathrm{J} 7.7,1.4,0.6, \mathrm{H} 5)$, $7.65(1 \mathrm{H}, \mathrm{td}, J$ J 7.7 1.4, H3), $7.57-7.49(5 \mathrm{H}, \mathrm{m}, \mathrm{H} 4, \mathrm{H} 2 \mathrm{a}, \mathrm{H} 3 \mathrm{a}), 7.45(1 \mathrm{H}, \mathrm{td}, J$ 7.6, 1.2, H2), $4.55(2 \mathrm{H}, \mathrm{s}$, $\mathrm{CH}_{2} \mathrm{Br}$ ). ${ }^{13} \mathrm{C}$ NMR $\left(126 \mathrm{MHz}, \mathrm{CDCl}_{3}\right) \delta 144.7$ (ArC), $138.4(\mathrm{ArC}), 138.2(\mathrm{ArC}), 133.9(\mathrm{ArCH}), 133.0(\mathrm{ArCH})$, $130.1(\mathrm{ArCH}), 129.5(\mathrm{ArCH}), 129.3(\mathrm{ArCH}), 127.9(\mathrm{ArCH}), 118.6(\mathrm{ArC}), 111.3(\mathrm{CN}), 32.9\left(\mathrm{CH}_{2} \mathrm{Br}\right)$.

\section{Hydroamination products}

Benzyl 3-(iso-butylamino) piperidine-1-carboxylate 6a<smiles>CC(C)CNC1CCCCC1</smiles>

6a was synthesised using the general hydroamination method using benzyl 3,4-dihydropyridine$1(2 \mathrm{H}$ )-carboxylate (200 mM final concentration), iso-butylamine (1 M final concentration), TRIP thiol (100 mM final concentration) and photocatalyst (1 mM) in a 4:1 mixture of anhydrous toluene and DCM. The mixture was injected into two sequential reactors at a flow rate of $50 \mu \mathrm{Lmin}^{-1}\left(\mathrm{t}_{\text {res }} 65.2\right.$ 
minutes). An $8 \mathrm{~mL}$ aliquot was taken for analysis which, after acid-base extraction, was isolated as a colourless oil (171 mg, 37\%).

Large scale preparation of $\mathbf{6 a}$

6a was synthesised using the general hydroamination method using benzyl 3,4-dihydropyridine$1(2 \mathrm{H})$-carboxylate (200 mM final concentration), iso-butylamine (1 M final concentration), TRIP thiol (100 mM final concentration) and photocatalyst $(1 \mathrm{mM})$ in a 4:1 mixture of anhydrous toluene and DCM. The mixture was injected into five sequential reactors at a flow rate of $125 \mu \mathrm{Lmin}^{-1}\left(\mathrm{t}_{\text {res }} 65.2\right.$ minutes). A $45 \mathrm{~mL}$ aliquot was taken for analysis which, after acid-base extraction, was isolated as a colourless oil (929 mg, 35\%).

Spectral data matches that reported. ${ }^{3}{ }^{1} \mathrm{H}$ NMR $\left(501 \mathrm{MHz}, \mathrm{CDCl}_{3}\right) \delta_{\mathrm{H}} 7.43-7.28(5 \mathrm{H}, \mathrm{m}, \mathrm{Cbz}), 5.13(2 \mathrm{H}$, s, $\left.\mathrm{CbzCH}_{2}\right), 4.05(1 \mathrm{H}, \mathrm{br} \mathrm{s}, \mathrm{H} 2), 3.88(1 \mathrm{H}, \mathrm{s}, \mathrm{H} 6), 2.95\left(1 \mathrm{H}, \mathrm{br} \mathrm{s}, \mathrm{H6} 6^{\prime}\right), 2.81-2.61\left(1 \mathrm{H}, \mathrm{m}, \mathrm{H2} 2^{\prime}\right), 2.54(1 \mathrm{H}$, br s, H3), $2.45(2 \mathrm{H}, \mathrm{br} \mathrm{s}, \mathrm{H} 1 \mathrm{a}), 1.98-1.89(1 \mathrm{H}, \mathrm{m}, \mathrm{H} 4), 1.68(2 \mathrm{H}, \mathrm{br} \mathrm{s}, \mathrm{H} 2 \mathrm{a}+\mathrm{H} 5), 1.52-1.44\left(1 \mathrm{H}, \mathrm{m}, \mathrm{H} 5^{\prime}\right)$, $1.30\left(1 \mathrm{H}, \mathrm{br} \mathrm{s}, \mathrm{H} 4^{\prime}\right), 0.88(6 \mathrm{H}, \mathrm{d}, \mathrm{J} 6.7, \mathrm{H3a}) .{ }^{13} \mathrm{C} N M R\left(126 \mathrm{MHz}, \mathrm{CDCl}_{3}\right) \delta_{\mathrm{C}} 155.4(\mathrm{C}=0), 137.0(\operatorname{ArC})$, 128.5 (ArCH), $128.0(\mathrm{ArCH}), 127.8(\mathrm{ArCH}), 67.0\left(\mathrm{CbzCH}_{2}\right), 55.2(\mathrm{C} 1 \mathrm{a}), 54.0(\mathrm{C} 3), 49.6(\mathrm{C} 2), 44.5(\mathrm{C} 6)$, 31.5 (C4), 28.6 (C2a), 24.0 (C5), 23.4 (C5), 20.6 (C3a).

Benzyl 3-(iso-butylamino)pyrrolidine-1-carboxylate 6b

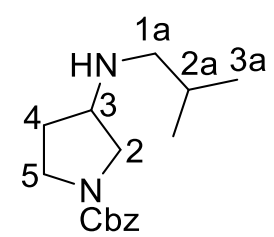

$\mathbf{6 b}$ was synthesised using the general hydroamination method using benzyl 2,3-dihydro- $1 \mathrm{H}$-pyrrole1- carboxylate (200 mM final concentration), iso-butylamine (1 M final concentration), TRIP thiol (100 mM final concentration) and photocatalyst $(1 \mathrm{mM})$ in a 4:1 mixture of toluene and DCM. The mixture was injected into a two sequential reactors at a flow rate of $50 \mu \mathrm{Lmin}^{-1}$ ( $\mathrm{t}_{\text {res }} 65.2$ minutes). A $4 \mathrm{~mL}$ aliquot was taken for analysis which after acid-base extraction gave $\mathbf{6 b}$ isolated as a colourless oil (110 mg, 50\%).

Spectral data matches that reported. ${ }^{4}{ }^{1} \mathrm{H}$ NMR $\left(501 \mathrm{MHz}, \mathrm{CDCl}_{3}\right) \delta_{\mathrm{H}} 7.42-7.26(5 \mathrm{H}, \mathrm{m}, \mathrm{Cbz}), 5.13$ $\left(2 \mathrm{H}, \mathrm{s}, \mathrm{CbzCH}_{2}\right), 3.67-3.58(1 \mathrm{H}, \mathrm{m}, \mathrm{H} 2), 3.60-3.49(1 \mathrm{H}, \mathrm{m}, \mathrm{H} 5), 3.41\left(1 \mathrm{H}, \mathrm{m}, \mathrm{H} 5^{\prime}\right), 3.31(1 \mathrm{H}, \mathrm{app} h, \mathrm{~J}$ 5.9, H3), $3.18(0.5 \mathrm{H}, \mathrm{dd}, \mathrm{J}$ 10.9, 5.3, H2'), $3.13(0.5 \mathrm{H}, \mathrm{dd}, \mathrm{J}$ 10.8, $5.7 \mathrm{H2}$ ) , 2.47-2.34 (2H, m, H1a), 2.12-2.00 (1H, m, H4), 1.79-1.61 (2H, m, H4' + H2a), $0.90(6 \mathrm{H}, \mathrm{d}, \mathrm{J} 6.8, \mathrm{H} 3 \mathrm{a}) .{ }^{13} \mathrm{C} N M R(126 \mathrm{MHz}$, $\left.\mathrm{CDCl}_{3}\right) \delta_{\mathrm{c}} 155.1(\mathrm{C}=\mathrm{O}), 137.2(\mathrm{ArC}), 128.6(\mathrm{ArCH}), 128.0(\mathrm{ArCH}) 66.8(\mathrm{CbzCH} 2), 58.0(\mathrm{C} 3), 57.1(\mathrm{C} 3)$, 
56.4 (C1a), 52.3 (C2), 52.0 (C2), 44.8 (C5), 44.5 (C5), 32.2 (C4), 31.5 (C4), 28.7 (C2a), 28.6 (C2a), 20.8 (C3a): 17 signals observed.

Benzyl 3-(iso-butylamino)azepane-1-carboxylate 6c

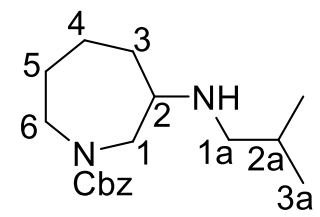

6c was synthesised using the general hydroamination method using benzyl 2,3,4,5-tetrahydro- $1 \mathrm{H}$ azepine-1-carboxylate ( $200 \mathrm{mM}$ final concentration), iso-butylamine (1 M final concentration), TRIP thiol (100 mM final concentration) and photocatalyst $(1 \mathrm{mM})$ in a 4:1 mixture of toluene and DCM. The mixture was injected into a two sequential reactors at a flow rate of $50 \mu \mathrm{Lmin}^{-1}\left(\mathrm{t}_{\text {res }} 65.2\right.$ minutes). An $8 \mathrm{~mL}$ aliquot was taken for analysis which after acid-base extraction gave $\mathbf{6 c}$ isolated as a colourless oil (58 $\mathrm{mg}, 12 \%)$.

Spectral data matches that reported. ${ }^{41} \mathrm{H}$ NMR $\left(501 \mathrm{MHz}, \mathrm{CD}_{3} \mathrm{OD}\right) \delta_{\mathrm{H}} 7.50-7.18(5 \mathrm{H}, \mathrm{m}, \mathrm{Cbz}), 5.28-$ $4.98\left(2 \mathrm{H}, \mathrm{m}, \mathrm{CbzCH}_{2}\right), 3.84(0.5 \mathrm{H}, \mathrm{dd}, J 14.4,4.2, \mathrm{H} 2), 3.75(0.5 \mathrm{H}, \mathrm{dd}, J 14.4,4.2, \mathrm{H} 2), 3.72-3.62$ (0.5H, m, H7), $3.50-3.43(1 \mathrm{H}, \mathrm{m}, \mathrm{H7}$ '), 3.25 (0.5H, dd, J 13.6, 5.4, H7), 2.96 (0.5H, dd, J 14.2, 9.4, $\left.H 2^{\prime}\right), 2.92-2.84\left(0.5 \mathrm{H}, \mathrm{m}, \mathrm{H} 2^{\prime}\right), 2.72-2.64(1 \mathrm{H}, \mathrm{m}), 2.64-2.51(1 \mathrm{H}, \mathrm{m}), 2.40-2.28(1 \mathrm{H}, \mathrm{m}), 2.27-$ $2.10(1 \mathrm{H}, \mathrm{m}), 1.97-1.76(2 \mathrm{H}, \mathrm{m}), 1.72-1.55(2 \mathrm{H}, \mathrm{m}), 1.41-1.27(2 \mathrm{H}, \mathrm{m}), 0.96(3 \mathrm{H}, \mathrm{d}, J 6.4), 0.81$ $\left(3 \mathrm{H}, \mathrm{t}, J\right.$ 6.2); ${ }^{13} \mathrm{C}$ NMR $\left(126 \mathrm{MHz}, \mathrm{CD}_{3} \mathrm{OD}\right) \delta_{\mathrm{c}} 157.0,156.4,136.8,128.3,128.2,128.0,127.9,127.8$, $127.51,127.50,67.2,67.0,58.3,54.7,54.4,49.6$, 49.0, 47.1, 47.0, 33.6, 32.2, 27.7, 27.6, 27.5, 26.8, $22.3,19.5,19.4 ; 28$ signals observed.

Benzyl 3-((2-ethoxy-2-oxoethyl)amino)piperidine-1-carboxylate 6d<smiles>CCOC(=O)CNC1CCSCC1</smiles>

6d was synthesised the general hydroamination method using benzyl 3,4-dihydropyridine-1(2H)carboxylate (200mM final concentration), glycine ethyl ester hydrochloride (0.4 M final concentration), lithium hydroxide monohydrate ( $0.4 \mathrm{M}$ final concentration), TRIP thiol (100 mM final concentration) and photocatalyst (1 mM) in a 4:1 mixture of toluene and DCM. The mixture was 
vigorously stirred for 30 minutes prior to being taking up into syringe. The mixture was injected into two sequential reactors at a flow rate of $50 \mu \mathrm{Lmin}^{-1}$ ( $t_{\text {res }} 65.2$ minutes). A $6 \mathrm{~mL}$ aliquot was taken for analysis which after acid-base extraction gave $\mathbf{6} \mathbf{d}$ isolated as a colourless oil (58mg, $15 \%$ ).

${ }^{1} \mathrm{H}$ NMR $\left(501 \mathrm{MHz}, \mathrm{CDCl}_{3}\right) \delta_{\mathrm{H}} 7.45-7.26(5 \mathrm{H}, \mathrm{m}, \mathrm{Cbz}), 5.20-5.04\left(2 \mathrm{H}, \mathrm{m}, \mathrm{CbzCH}_{2}\right), 4.17(2 \mathrm{H}, \mathrm{q}, J 7.2$, $\left.\mathrm{OCH}_{2}\right), 4.08-3.89(1 \mathrm{H}, \mathrm{m}, \mathrm{H} 2), 3.85(1 \mathrm{H}, \mathrm{app} \mathrm{dt}, \mathrm{J} 13.3,4.3, \mathrm{H} 6), 3.54-3.30(2 \mathrm{H}, \mathrm{m}, \mathrm{H} 1 \mathrm{a}), 2.96$ (1 H, app ddd, J 13.6, 10.5, 3.2, H6'), $2.89-2.70\left(1 \mathrm{H}, \mathrm{m}, \mathrm{H} 2^{\prime}\right), 2.62-2.52(1 \mathrm{H}, \mathrm{m}, \mathrm{H} 3), 1.97-1.86(1 \mathrm{H}$, m, H4), $1.76-1.66(1 \mathrm{H}, \mathrm{m}, \mathrm{H} 5), 1.52-1.42\left(1 \mathrm{H}, \mathrm{m}, \mathrm{H} 5^{\prime}\right), 1.39-1.28\left(1 \mathrm{H}, \mathrm{m}, \mathrm{H} 6^{\prime}\right), 1.26(1 \mathrm{H}, \mathrm{t}, \mathrm{J} 7.2$, $\mathrm{CH} 3) .{ }^{13} \mathrm{C}$ NMR (126 MHz, CDCl 3 ) $\delta 172.6$ (C2a), $155.5(\mathrm{C}=\mathrm{O}), 136.9,(\mathrm{ArC}) 128.6(\mathrm{ArCH}), 128.1(\mathrm{ArCH})$, 128.0 (ArCH), 127.9 ( $\mathrm{ArCH}), 67.2\left(\mathrm{CbzCH}_{2}\right), 61.0\left(\mathrm{OCH}_{2}\right), 53.6(\mathrm{C} 3), 53.5(\mathrm{C} 3), 49.2$ (C6), 48.5 (C1a), 44.7 (C2), 44.5 (C2), 31.3 (C5), 23.7 (C4), 23.3 (C4), $14.3\left(\mathrm{CH}_{3}\right)$; 19 signals observed.

tert-Butyl 4-(1-((benzyloxy)carbonyl)piperidin-3-yl)piperazine-1-carboxylate 6e<smiles>CC(C)(C)OC(=O)N1CCN(C2CCCCC2)CC1</smiles>

6e was synthesised using the general hydroamination method using benzyl 3,4-dihydropyridine1(2H)- carboxylate (200 mM final concentration), 1-Boc piperazine (0.4 M final concentration), TRIP thiol (100 mM final concentration) and photocatalyst $(1 \mathrm{mM})$ in a 4:1 mixture of toluene and DCM. The mixture was injected into a two sequential reactors at a flow rate of $50 \mu \mathrm{Lmin}^{-1}\left(\mathrm{t}_{\text {res }} 65.2\right.$ minutes). An $8 \mathrm{~mL}$ aliquot was taken for analysis which after purification by column chromatography (20\%-50\% ethyl acetate in hexane) gave $6 \mathrm{~d}$ isolated as a colourless oil (180 mg, $28 \%$ ).

${ }^{1} \mathrm{H}$ NMR $\left(501 \mathrm{MHz}, \mathrm{CDCl}_{3}\right) \delta_{\mathrm{H}} 7.41-7.28(5 \mathrm{H}, \mathrm{m}, \mathrm{Cbz}), 5.12\left(2 \mathrm{H}, \mathrm{s}, \mathrm{CbzCH}_{2}\right), 4.32-4.08(1 \mathrm{H}, \mathrm{m}, \mathrm{H} 6)$, $4.00(1 \mathrm{H}, \mathrm{br} \mathrm{s}, \mathrm{H} 2), 3.41(4 \mathrm{H}, \mathrm{br} \mathrm{s}, \mathrm{H} 3 \mathrm{a}), 2.78(2 \mathrm{H}, \mathrm{br} \mathrm{s}, \mathrm{H} 2+\mathrm{H} 6), 2.52(4 \mathrm{H}, \mathrm{s}, \mathrm{H} 2 \mathrm{a}), 2.33(1 \mathrm{H}, \mathrm{s}, \mathrm{H} 3)$, $1.96(1 \mathrm{H}, \mathrm{br} \mathrm{s}, \mathrm{H} 5), 1.75(1 \mathrm{H}, \mathrm{br} \mathrm{s}, \mathrm{H} 4), 1.45\left(11 \mathrm{H}, \mathrm{br} \mathrm{s}, \mathrm{H} 4^{\prime}+\mathrm{H} 5^{\prime}+\mathrm{Boc}\right) ;{ }^{13} \mathrm{C} \mathrm{NMR}\left(126 \mathrm{MHz}, \mathrm{CDCl}_{3}\right) \delta$ $155.5(\mathrm{C}=\mathrm{O}), 154.8(\mathrm{C}=\mathrm{O}), 137.0(\mathrm{ArC}), 128.6(\mathrm{ArCH}), 128.2(\mathrm{ArCH}), 128.0(\mathrm{ArCH}), 79.9(\mathrm{OBoc}), 67.3$ ( $\mathrm{CbzCH} 2$ ), 60.5 (C3), 49.5 (C2a), 46.6 (C2), 44.6 (C6), 44.3 (C3a), 28.5 (Boc), 27.6 (C4/C5), 27.1(C4/C5), 24.5(C4/C5), 24.3(C4/C5); 19 signals observed. 
Benzyl 3-(4-(tert-butoxycarbonyl)piperazin-1-yl)azepane-1-carboxylate $6 \mathbf{f}$

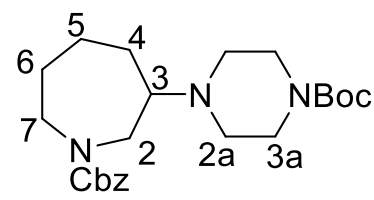

6f was synthesised using the general hydroamination method using benzyl 2,3,4,5-tetrahydro- $1 \mathrm{H}-$ azepine-1-carboxylate (200 mM final concentration), 1-Boc piperazine (0.4 M final concentration), TRIP thiol (100 mM final concentration) and photocatalyst (1 $\mathrm{mM}$ ) in a 4:1 mixture of toluene and DCM. The mixture was injected into two sequential reactors at a flow rate of $50 \mu \mathrm{Lmin}^{-1}\left(\mathrm{t}_{\text {res }} 65.2\right.$ minutes). An $8 \mathrm{~mL}$ aliquot was taken for analysis which after purification by column chromatography (20\%-50\% ethyl acetate in hexane) gave $6 f$ isolated as a colourless oil ( $86 \mathrm{mg}, 13 \%$ )

${ }^{1} \mathrm{H}$ NMR $\left(501 \mathrm{MHz}, \mathrm{CDCl}_{3}\right) \delta_{\mathrm{H}} 7.43-7.29(5 \mathrm{H}, \mathrm{m}), 5.18-5.08(2 \mathrm{H}, \mathrm{m}), 3.85-3.73(1 \mathrm{H}, \mathrm{m}), 3.65-3.56$ $(0.5 \mathrm{H}, \mathrm{m}), 3.53-3.21(5.5 \mathrm{H}, \mathrm{m}), 3.12-3.00(0.5 \mathrm{H}, \mathrm{m}), 2.82-2.78(2.5 \mathrm{H} . \mathrm{m}), 2.55-2.38(2 \mathrm{H}, \mathrm{m}), 1.94-$ $1.72(4 \mathrm{H}, \mathrm{m}), 1.59-1.52(2 \mathrm{H}, \mathrm{m}), 1.47-1.42(9 \mathrm{H}, \mathrm{m}), 1.35-1.24(1 \mathrm{H}, \mathrm{m}) ; 13 \mathrm{C} \mathrm{NMR}(126 \mathrm{MHz}$, $\left.\mathrm{CDCl}_{3}\right) \delta 156.3,155.9,154.6,136.8,136.7,128.5,128.2,128.2,128.0,127.8,79.9,79.7,67.3,67.2$, $65.0,64.9,48.9,47.5,47.3,47.1,30.1,29.4,28.43,28.42,27.8,27.3,24.5,24.0 ; 28$ signals observed. 
${ }^{1} \mathrm{H}$ and ${ }^{13} \mathrm{C}$ NMR Spectra of Products

2a (90\% pure, contains 10\% 1,1-dibromide)
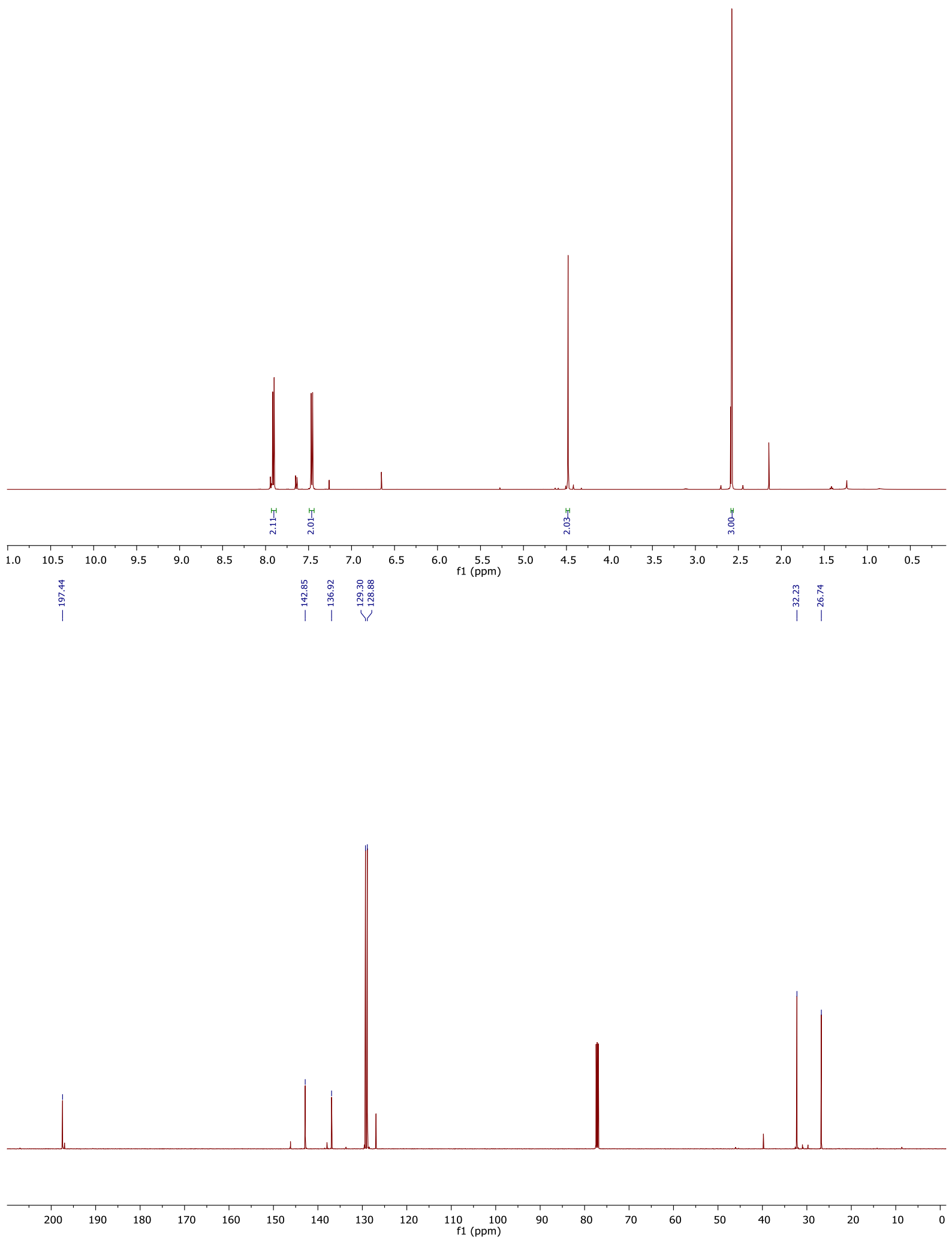

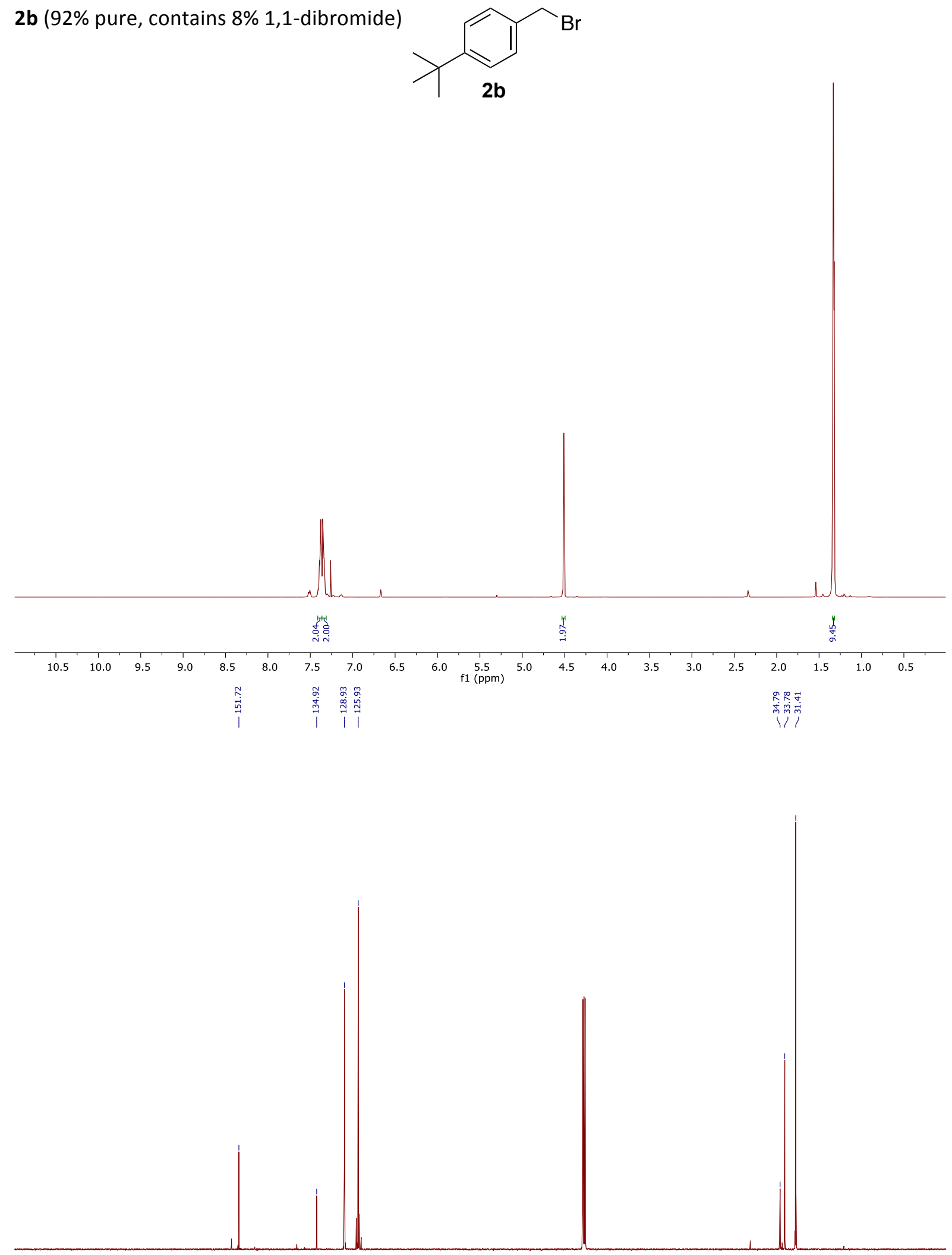

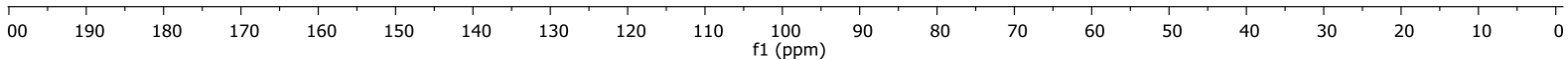


2c (93\% pure, contains 7\% $p$-anisaldehyde)
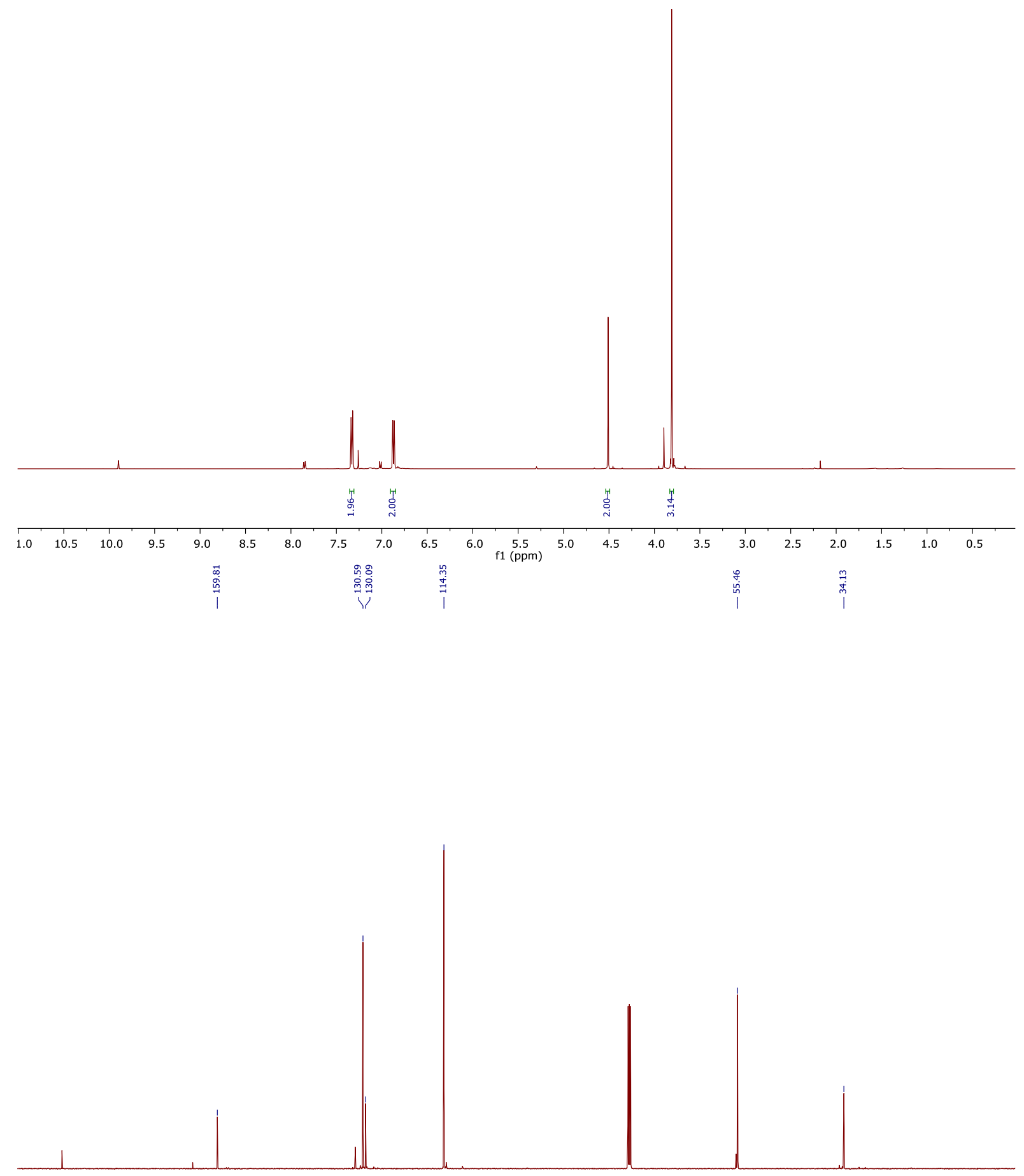

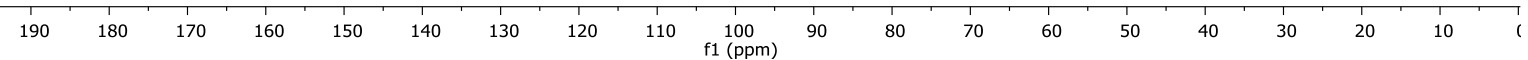


2d (94\% pure, contains 6\% 1,1-dibromide)
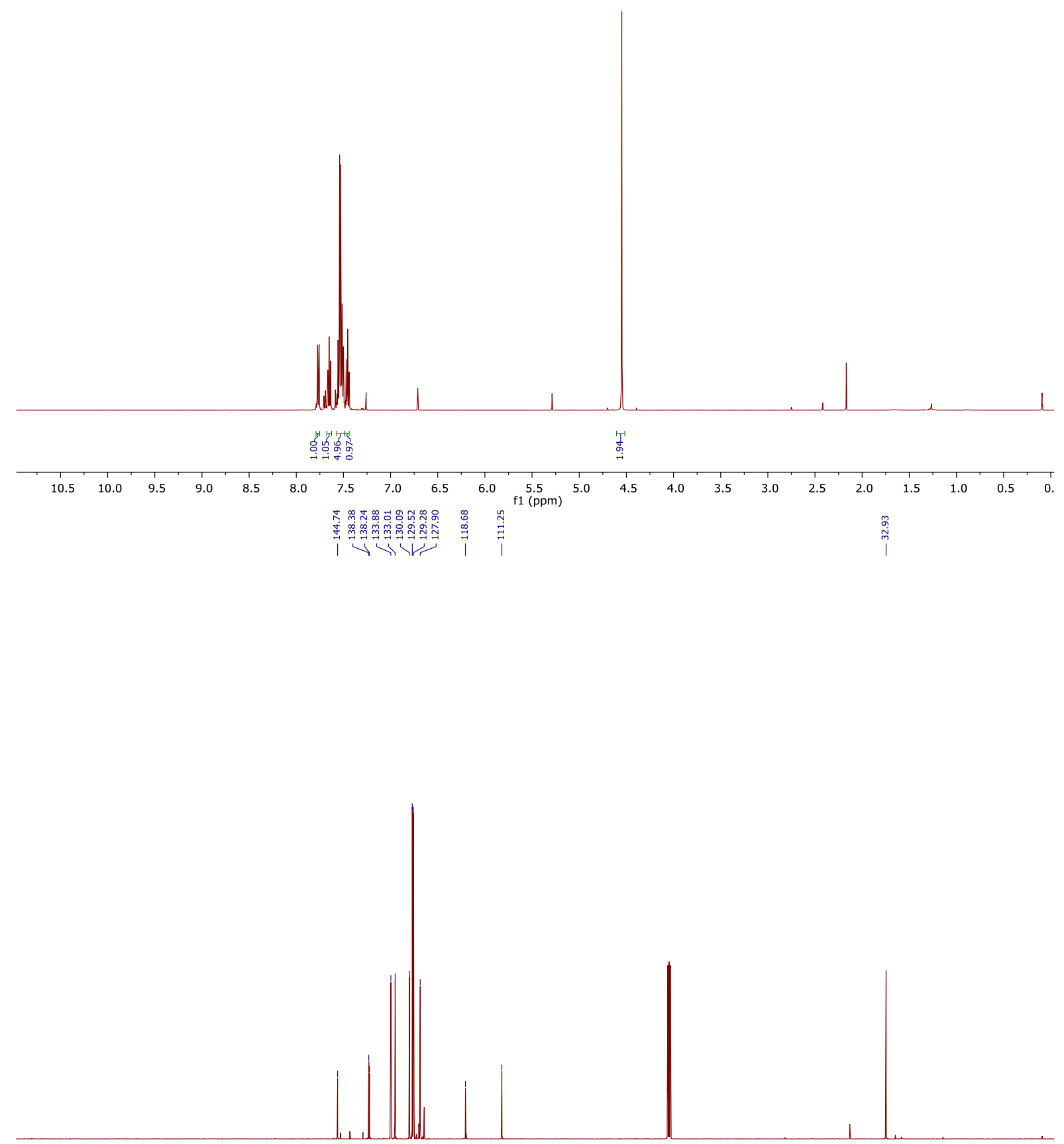

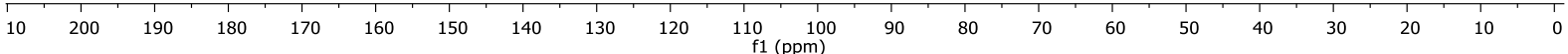



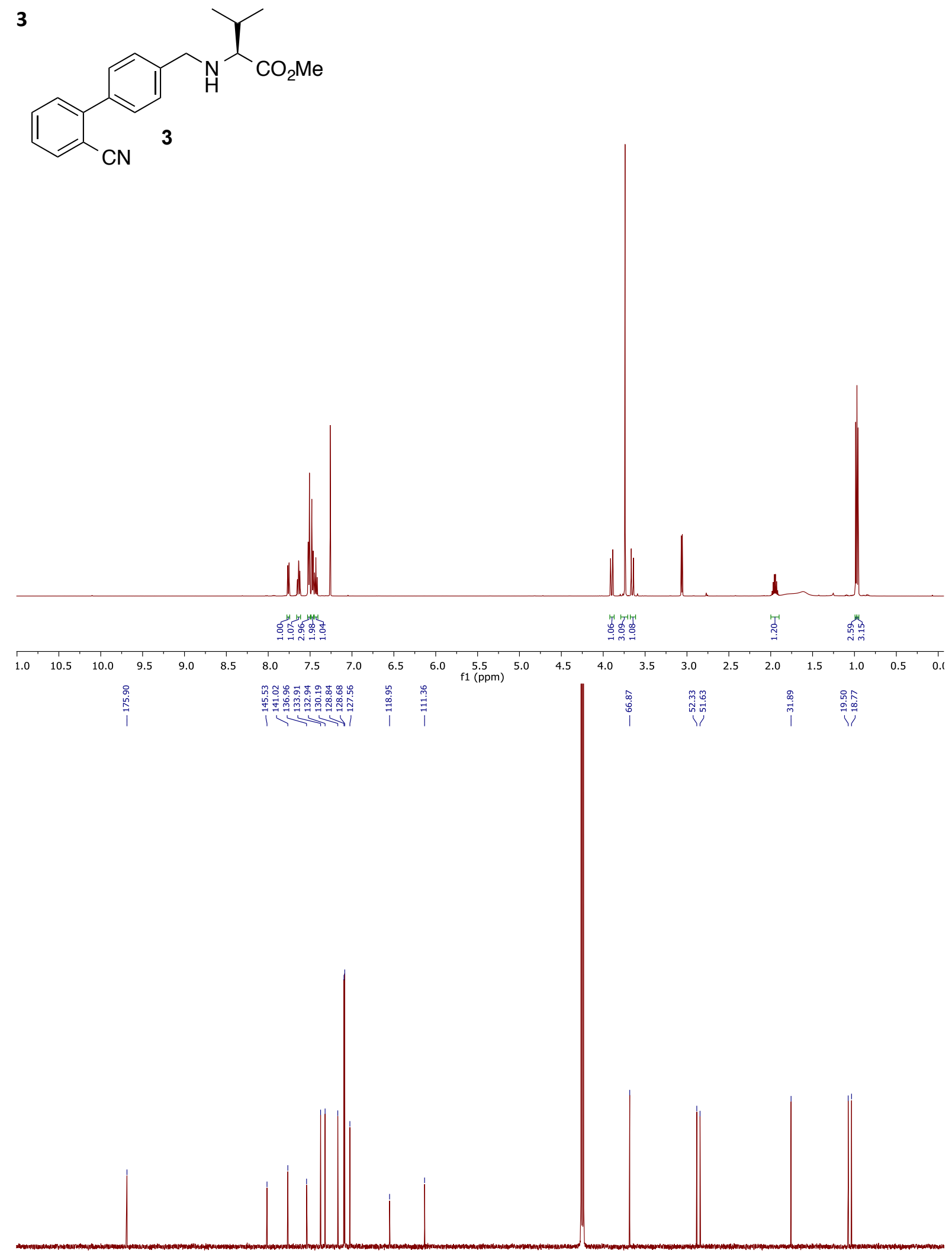

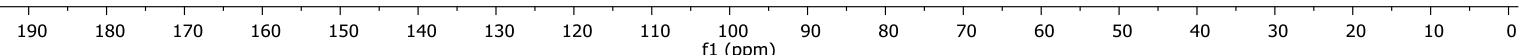



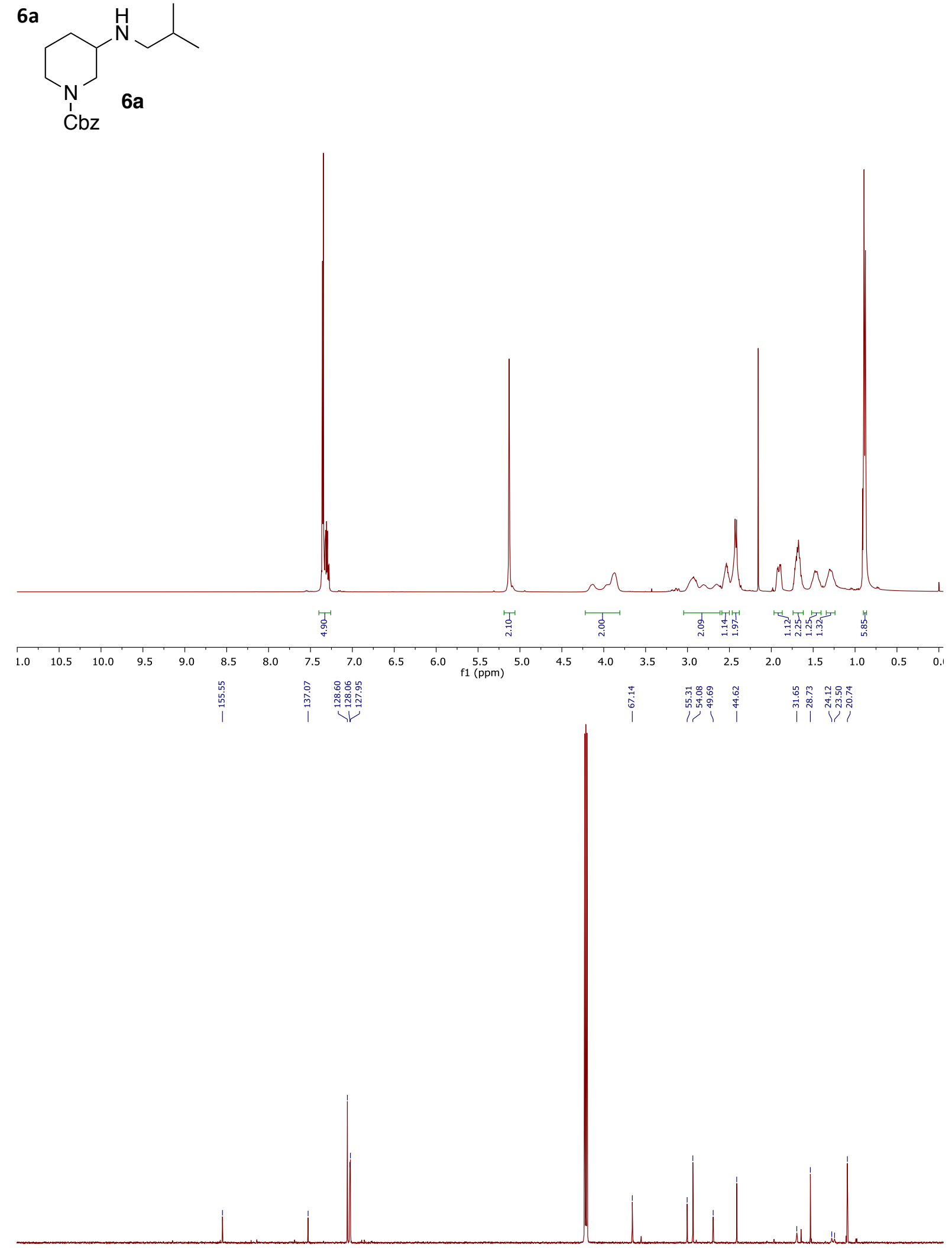

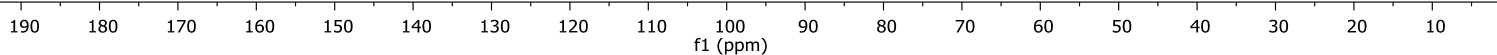



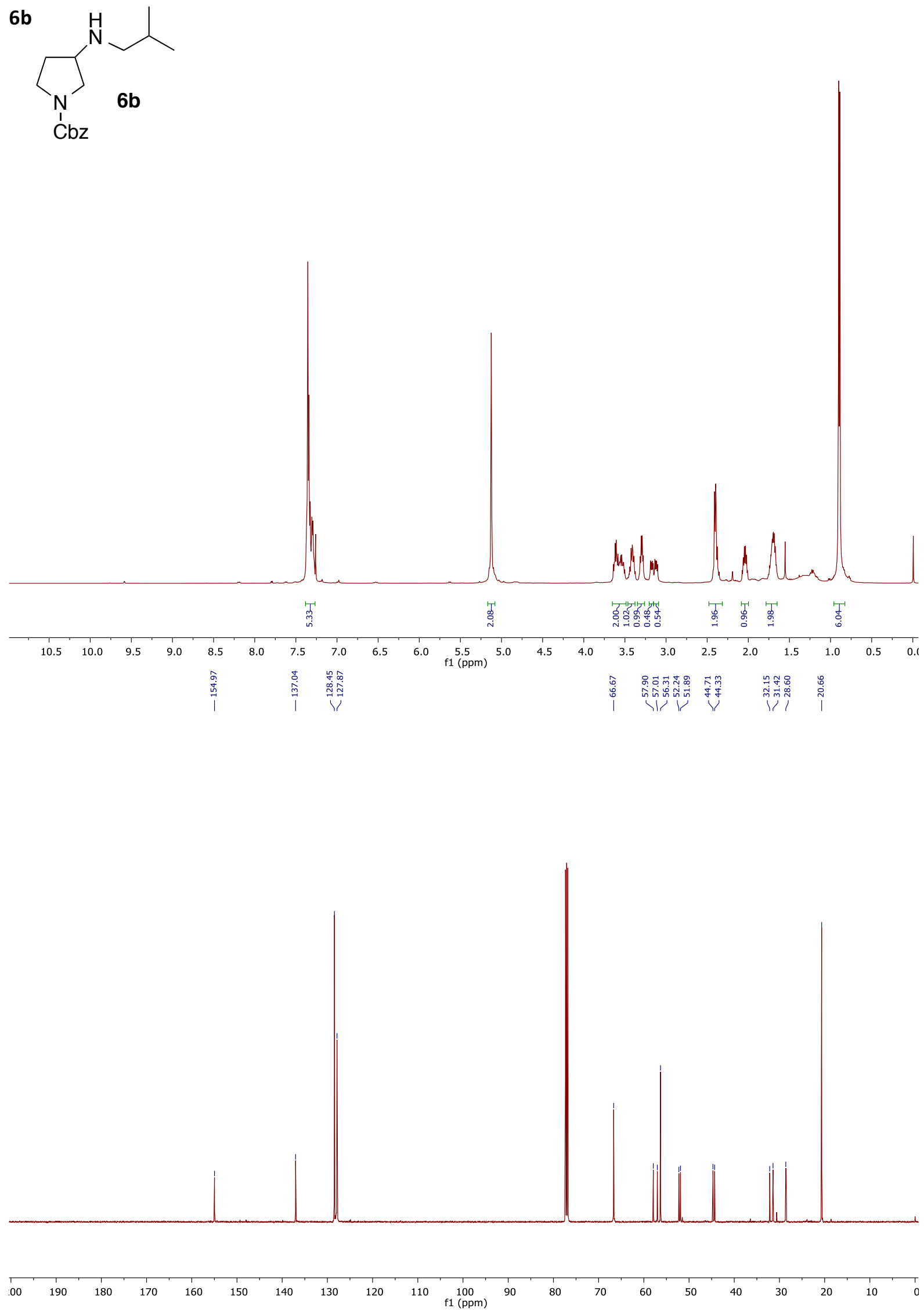

18 

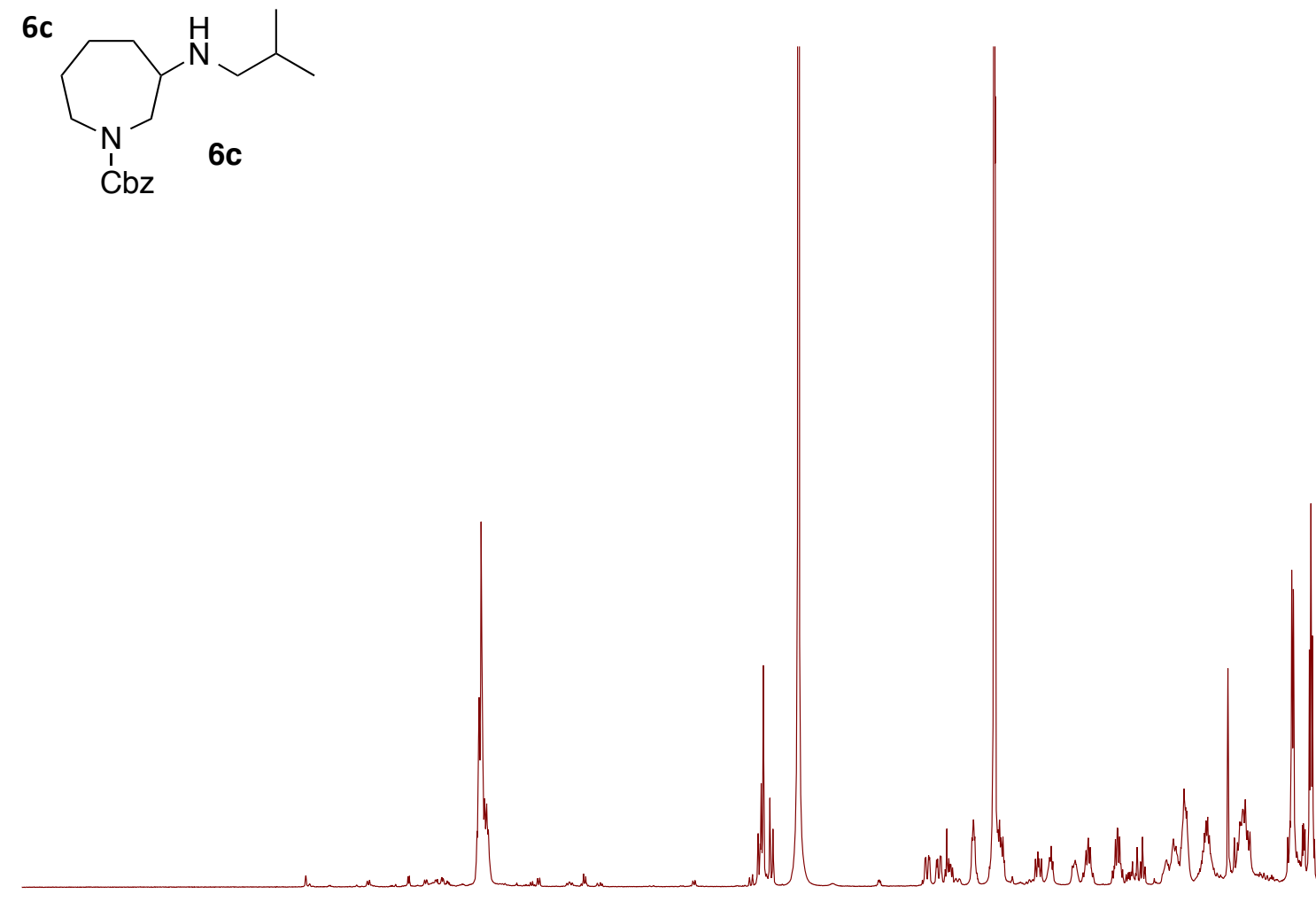

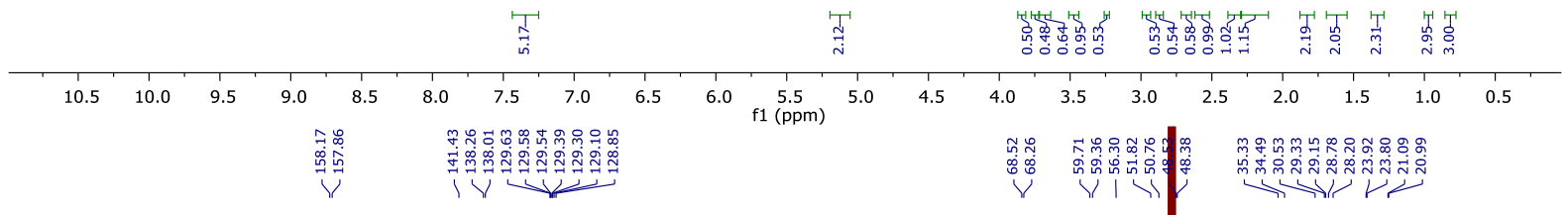

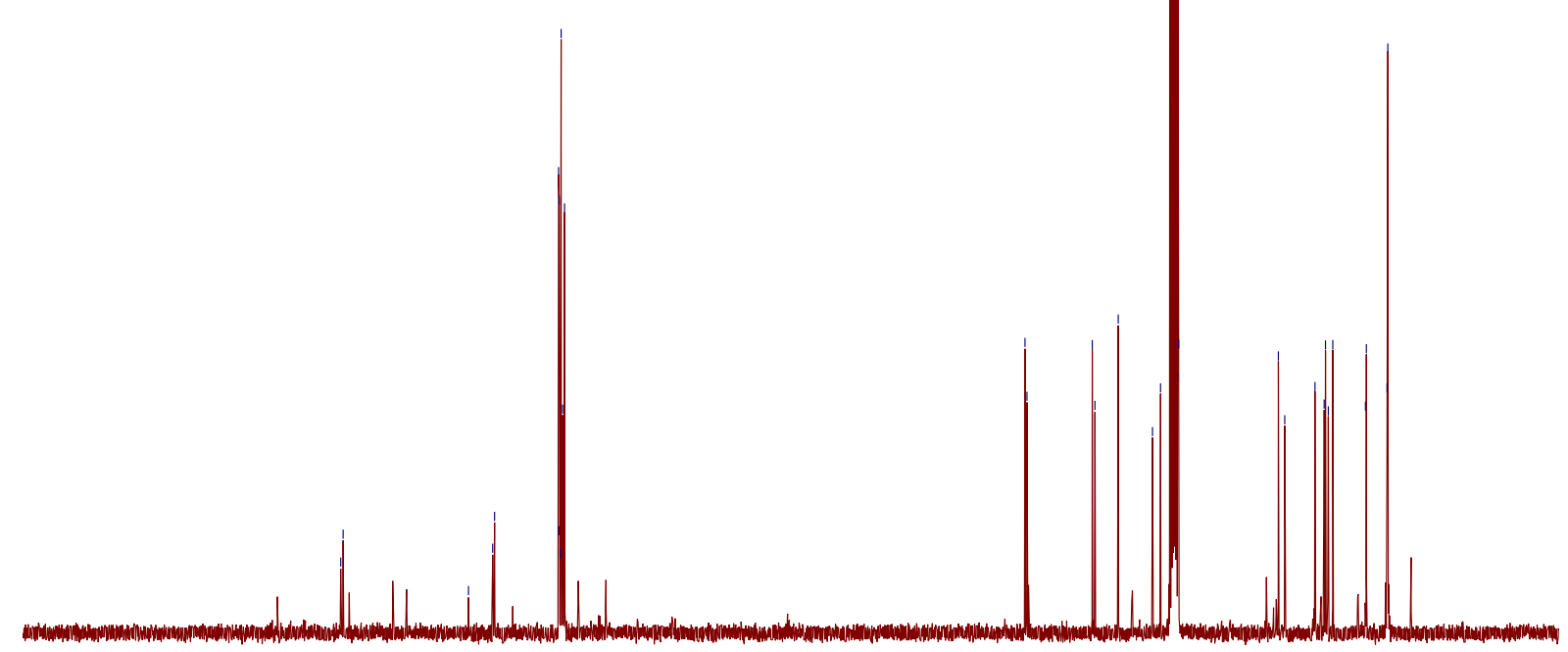

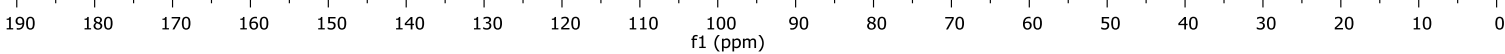


<smiles>CCOC(=O)CNC1CCCN(C(=O)OCc2ccccc2)C1</smiles>

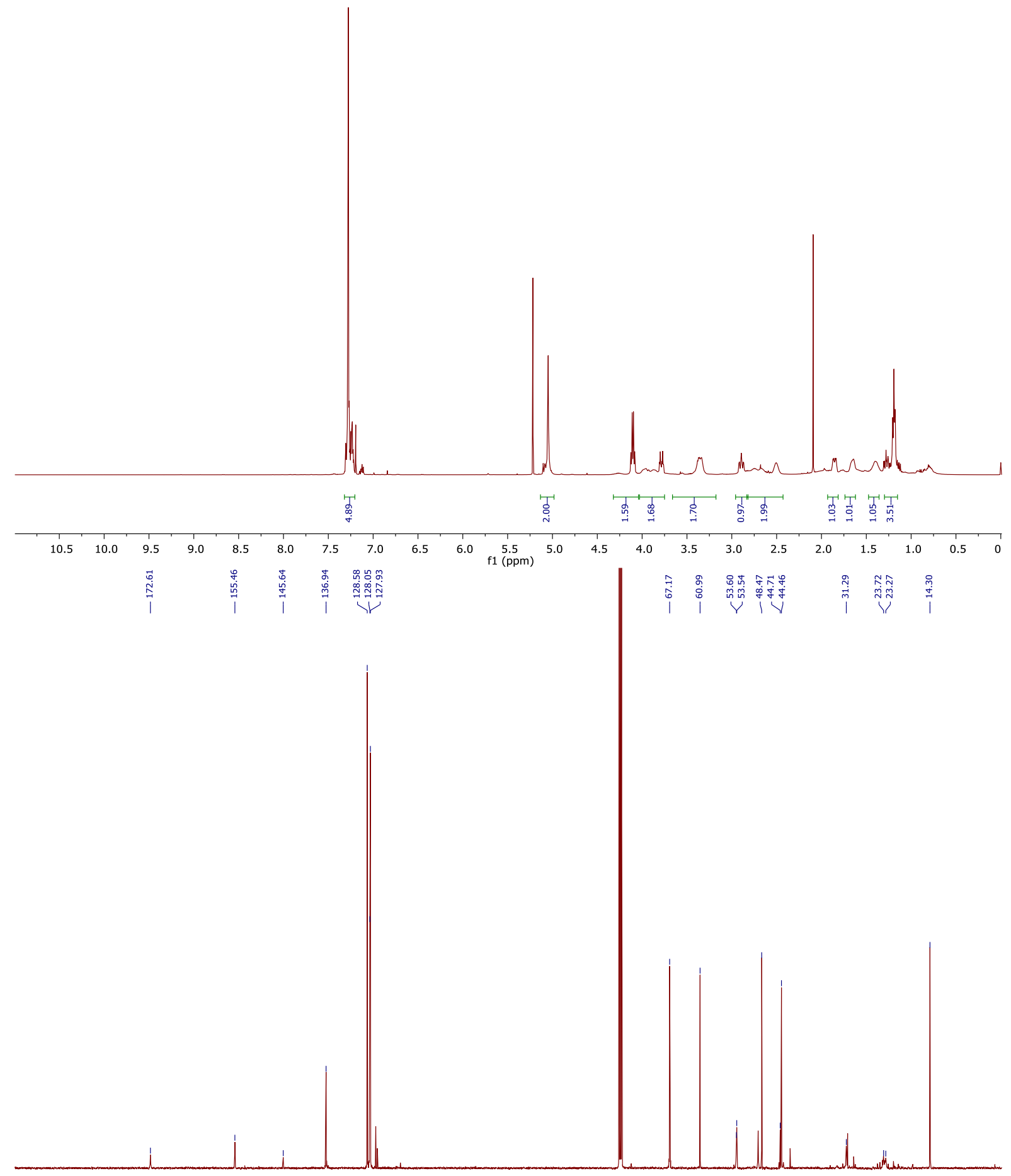

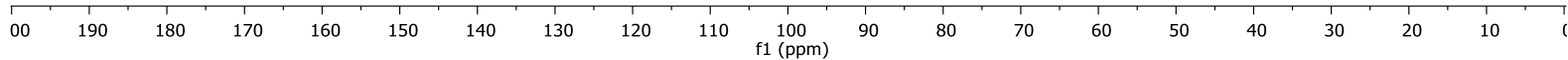




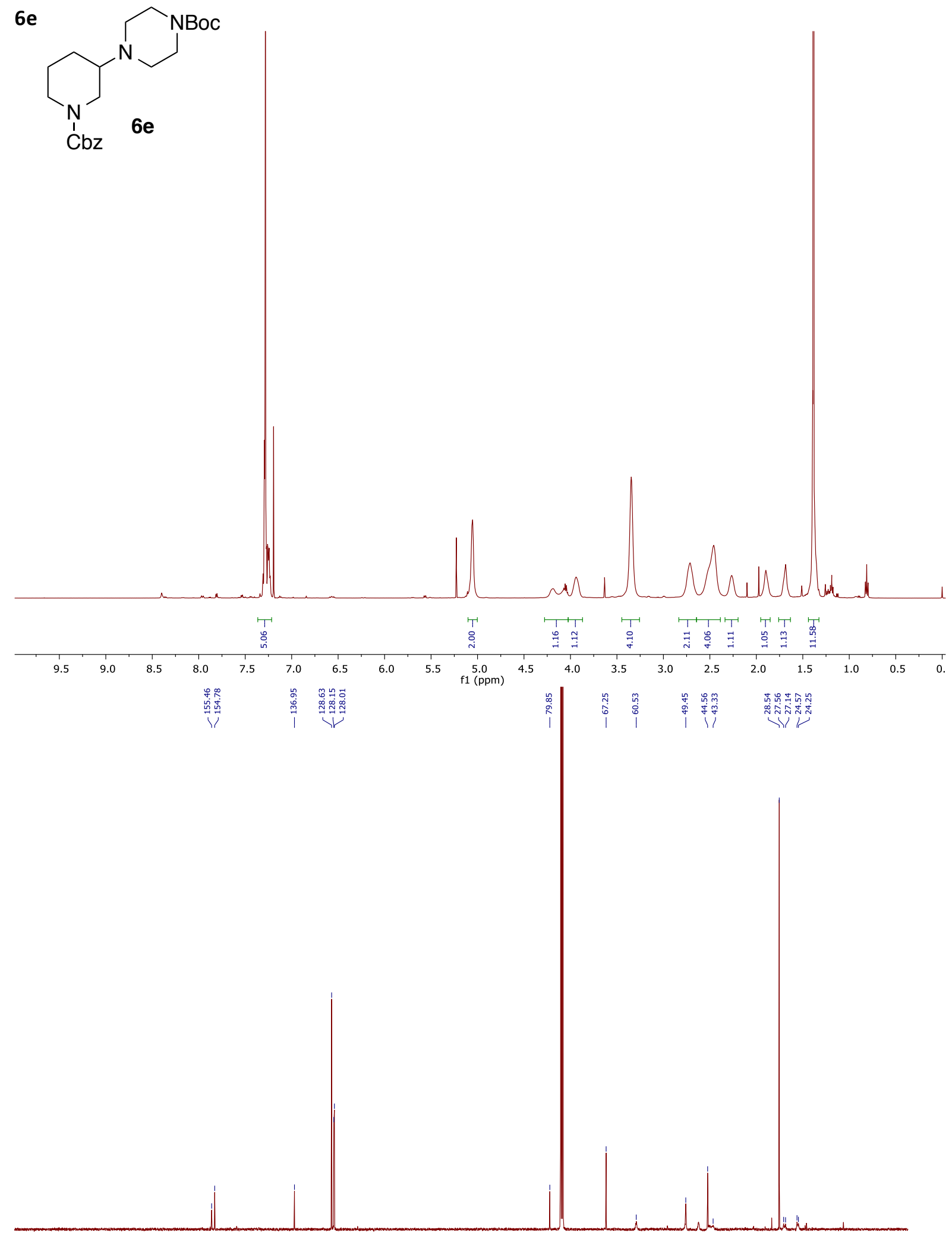

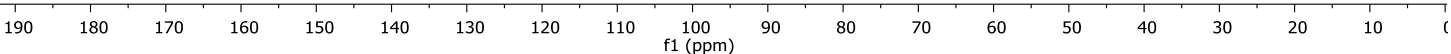




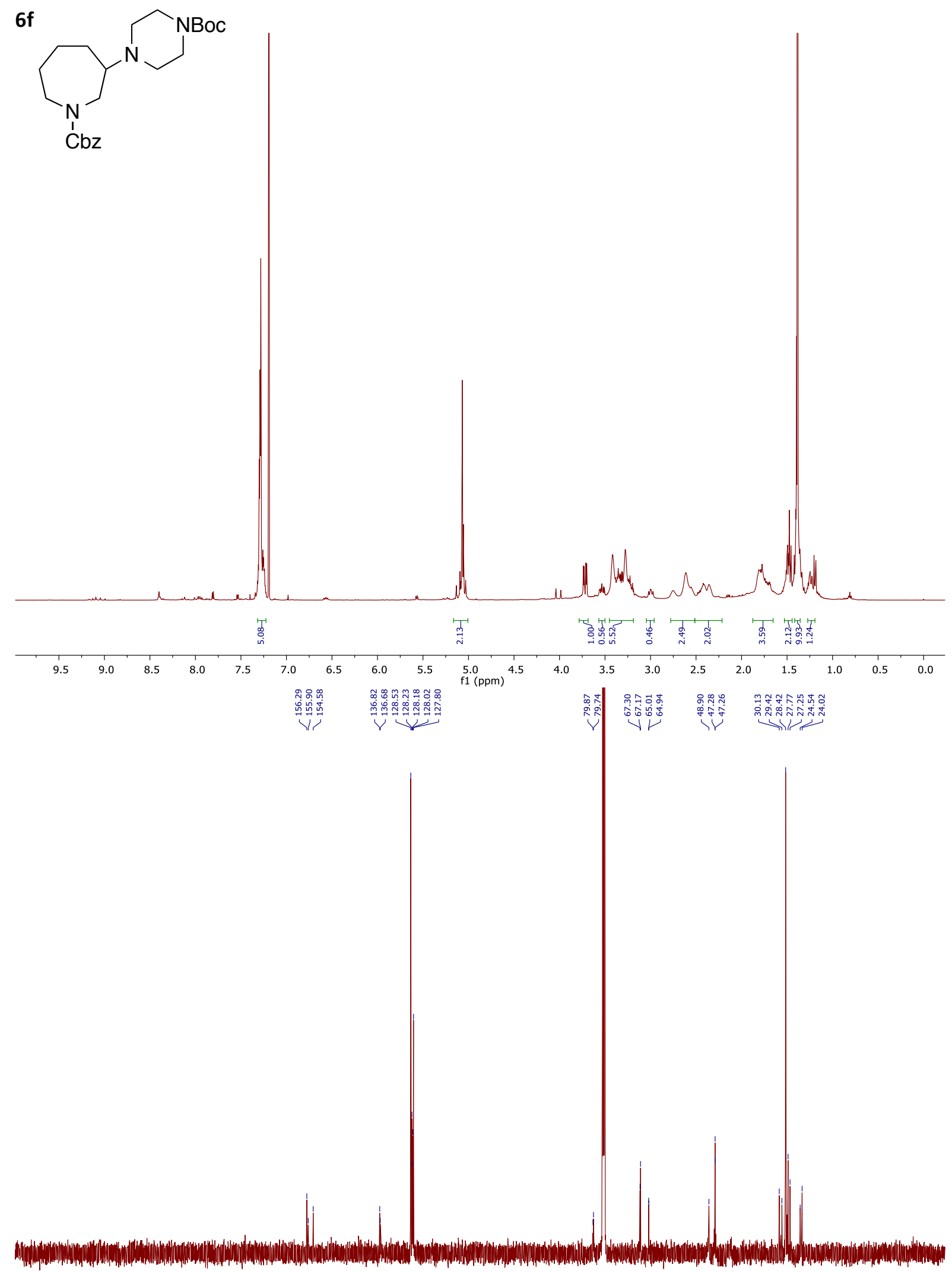

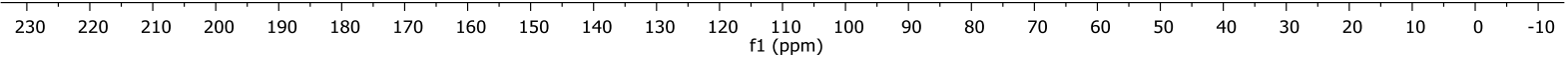




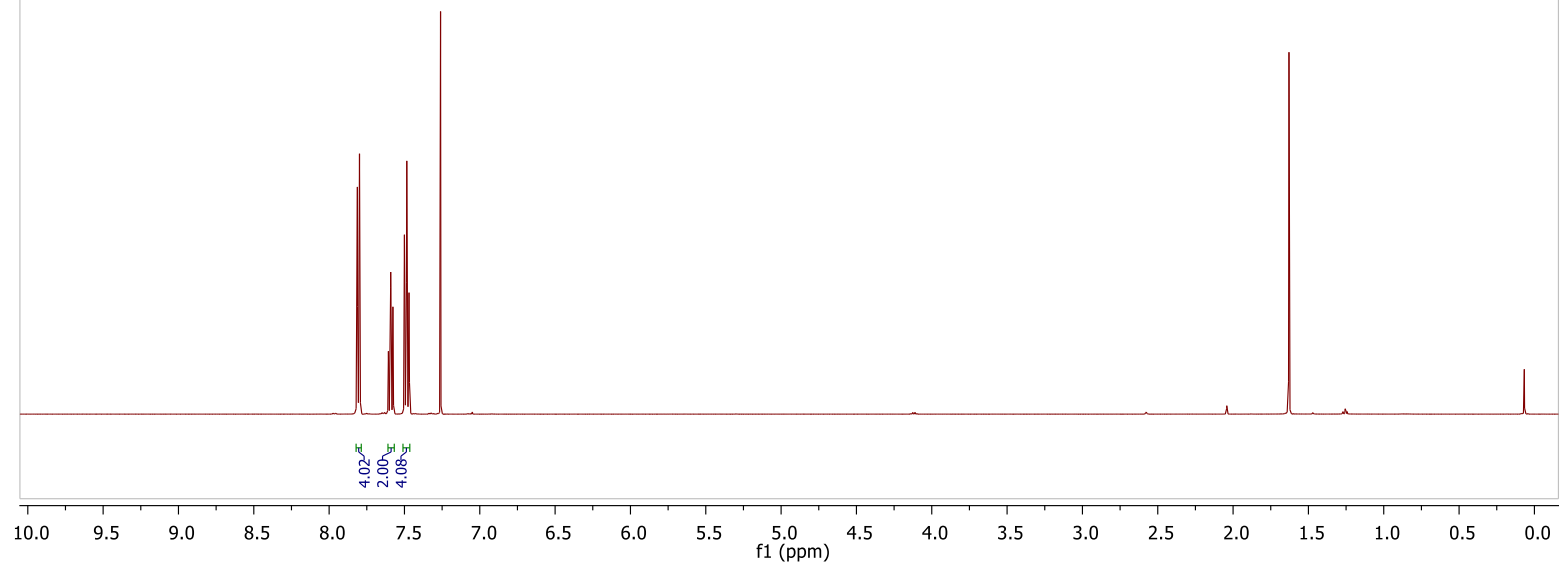

|

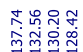

रे।
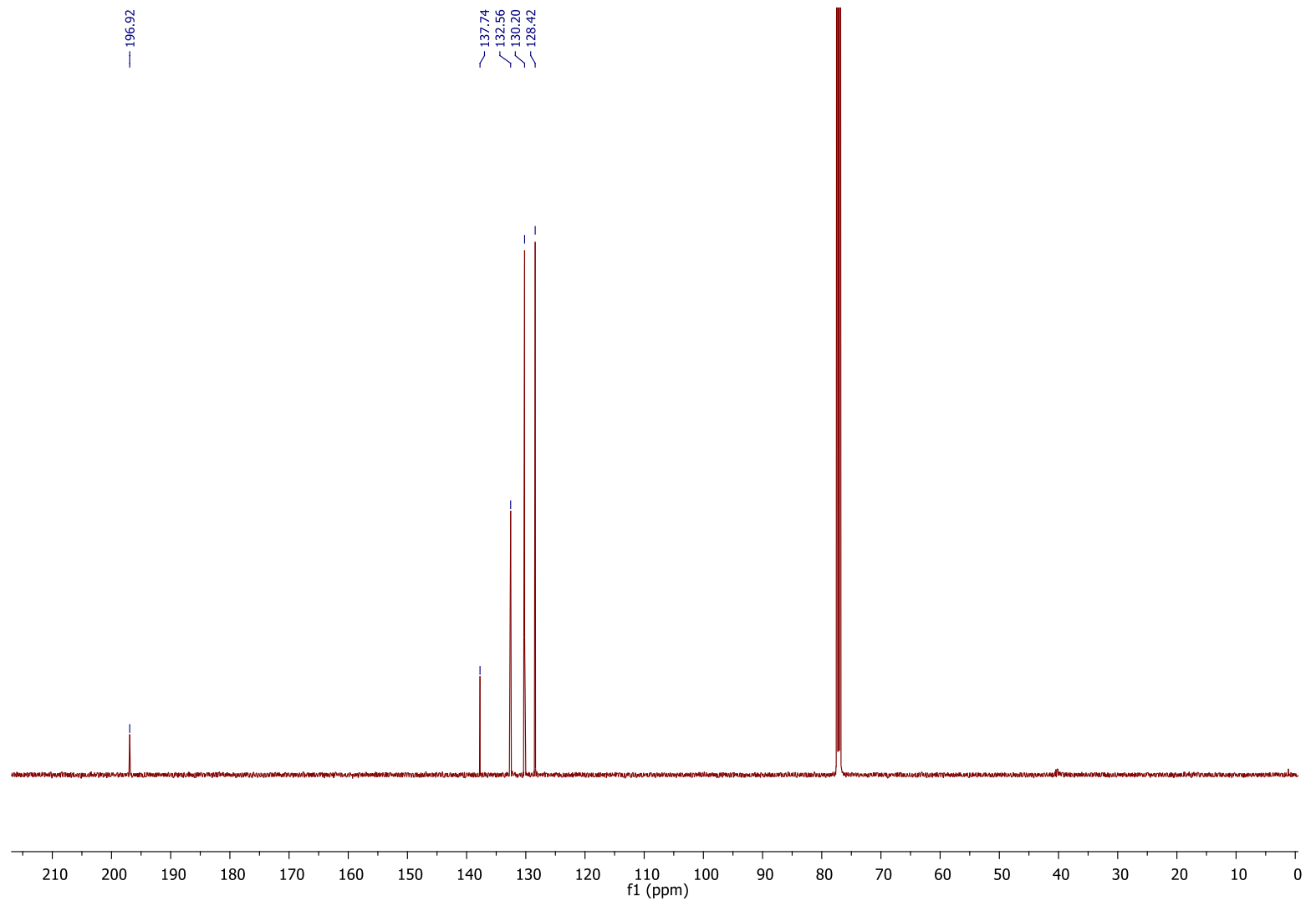
Crude NMR for conversion/selectivity for $2 \mathrm{a}$. Top, $0.5 \mathrm{M}$ reaction; bottom, $2 \mathrm{M}$ reaction

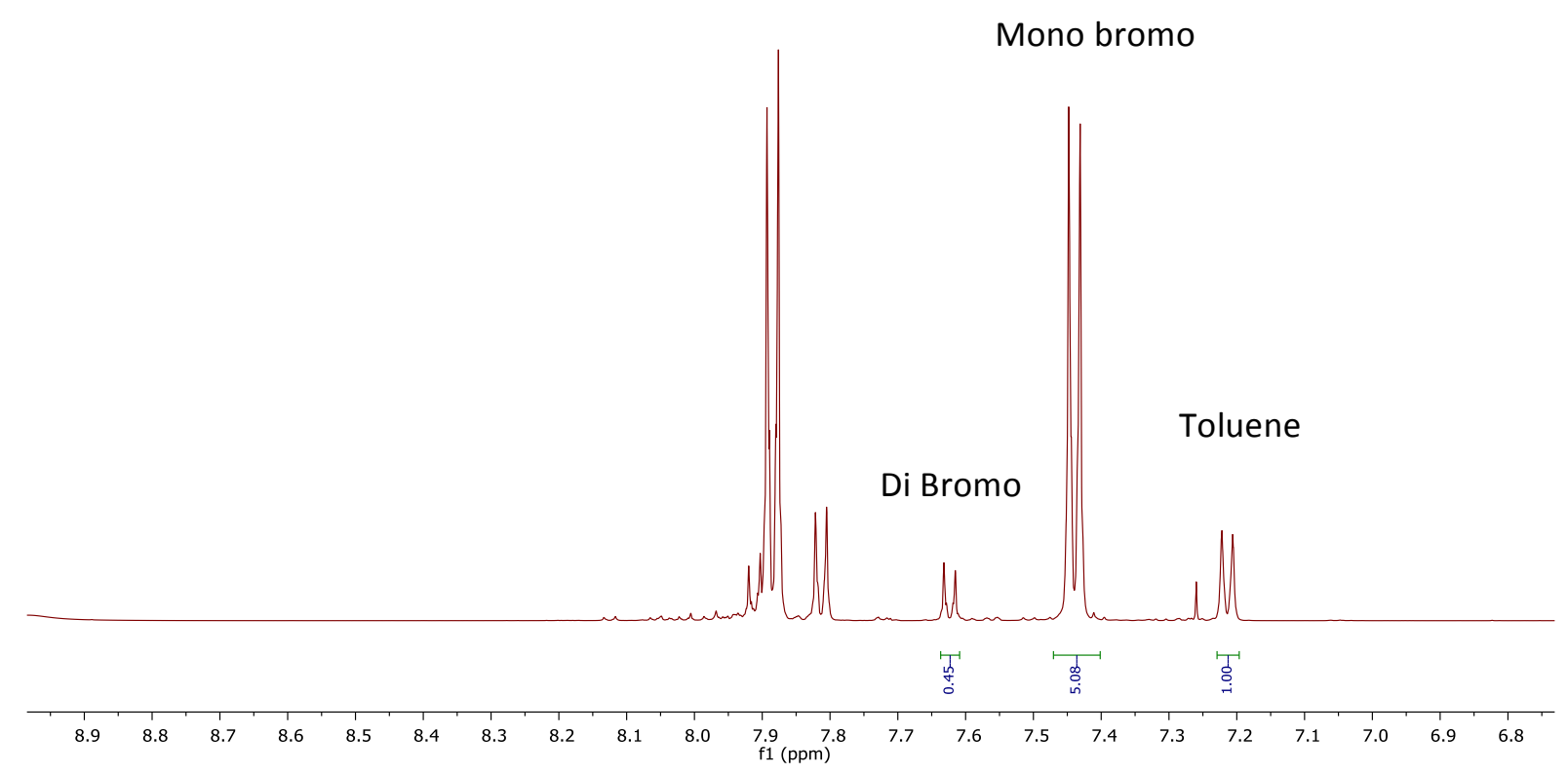

Conversion $=85 \%$, selectivity $=92 \%$

\section{Mono bromo}

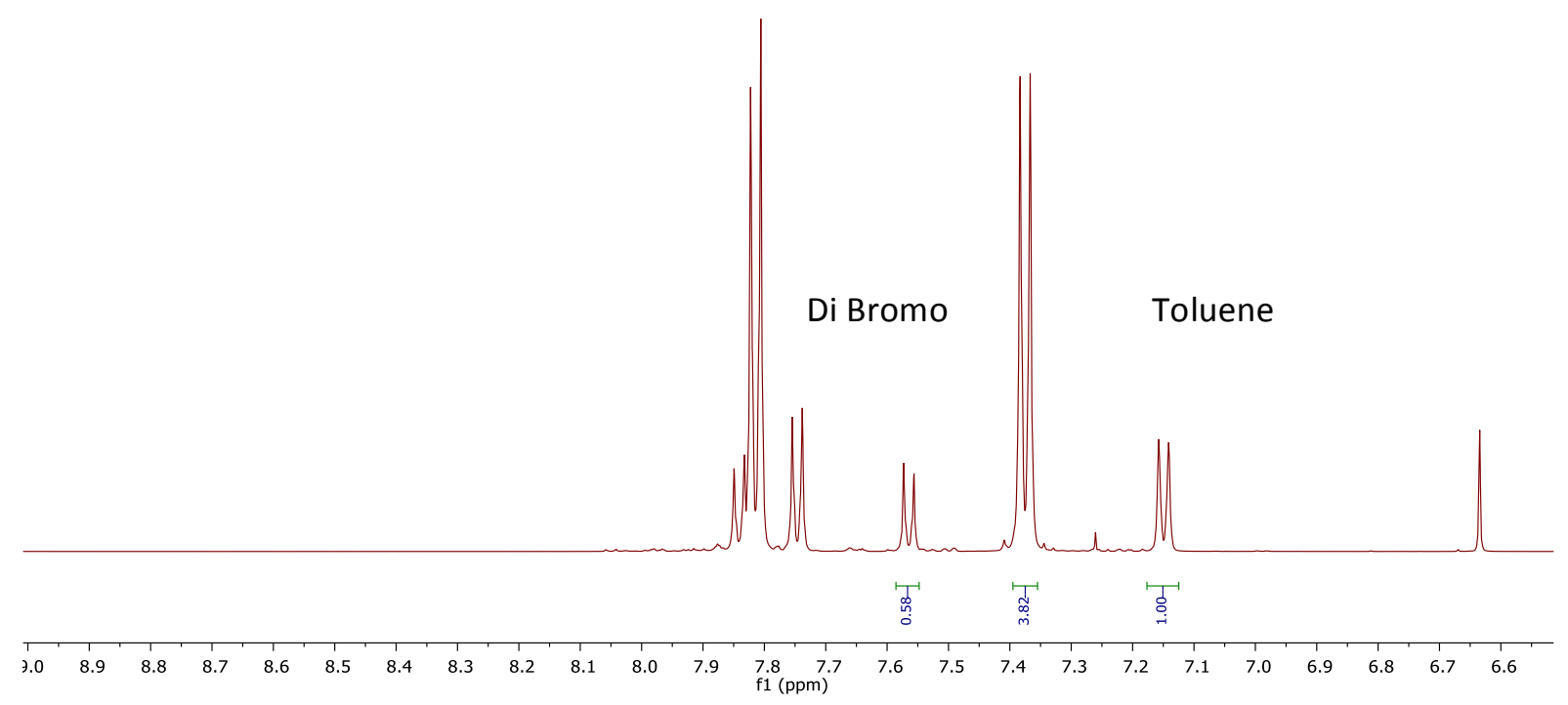

Conversion $=81 \%$, selectivity $=87 \%$ 
Crude NMR for conversion/selectivity for $\mathbf{2 b}$. Top, $0.5 \mathrm{M}$ reaction; bottom, $2 \mathrm{M}$ reaction

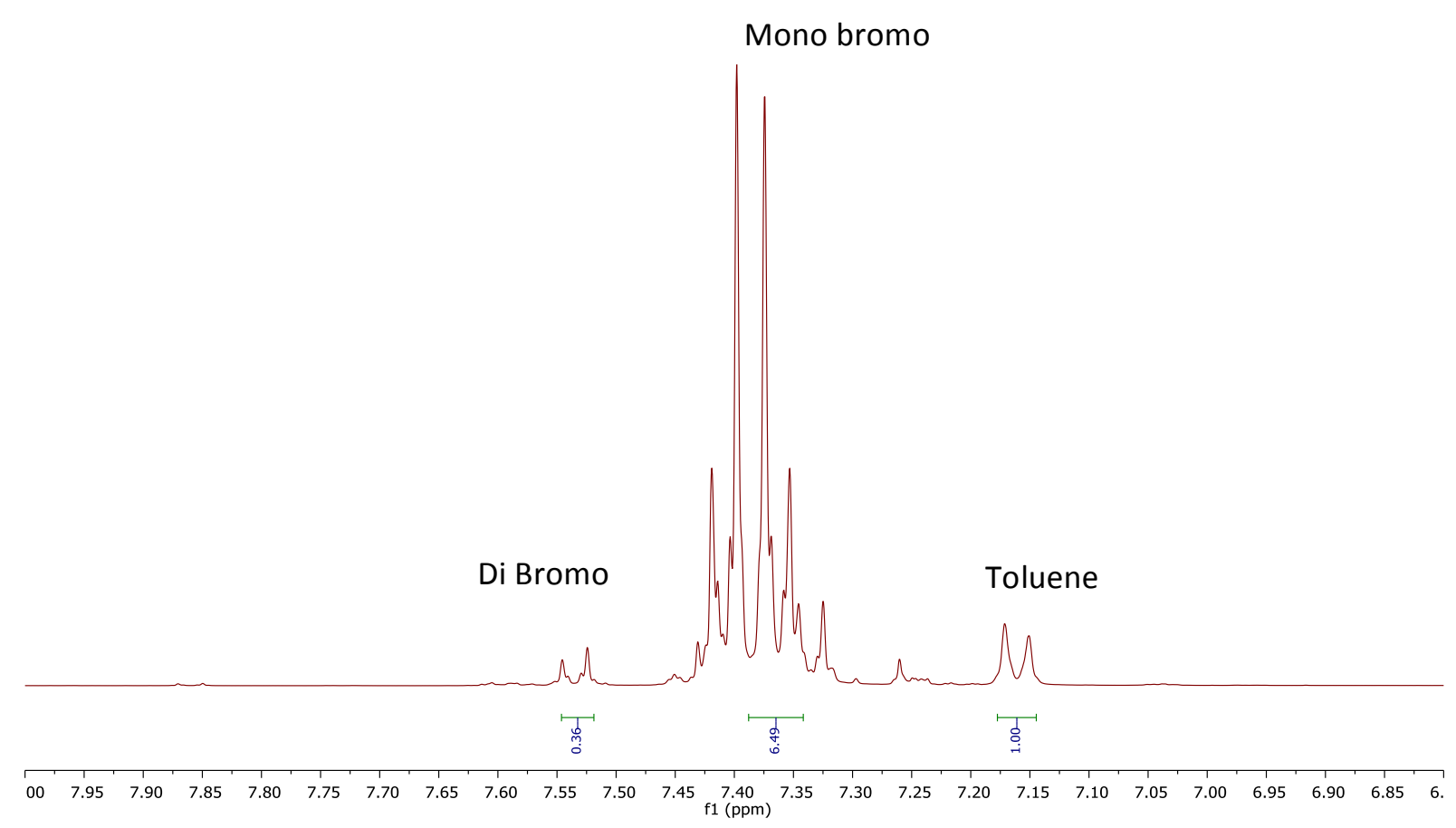

Conversion $=87 \%$, selectivity $=95 \%$

Mono bromo

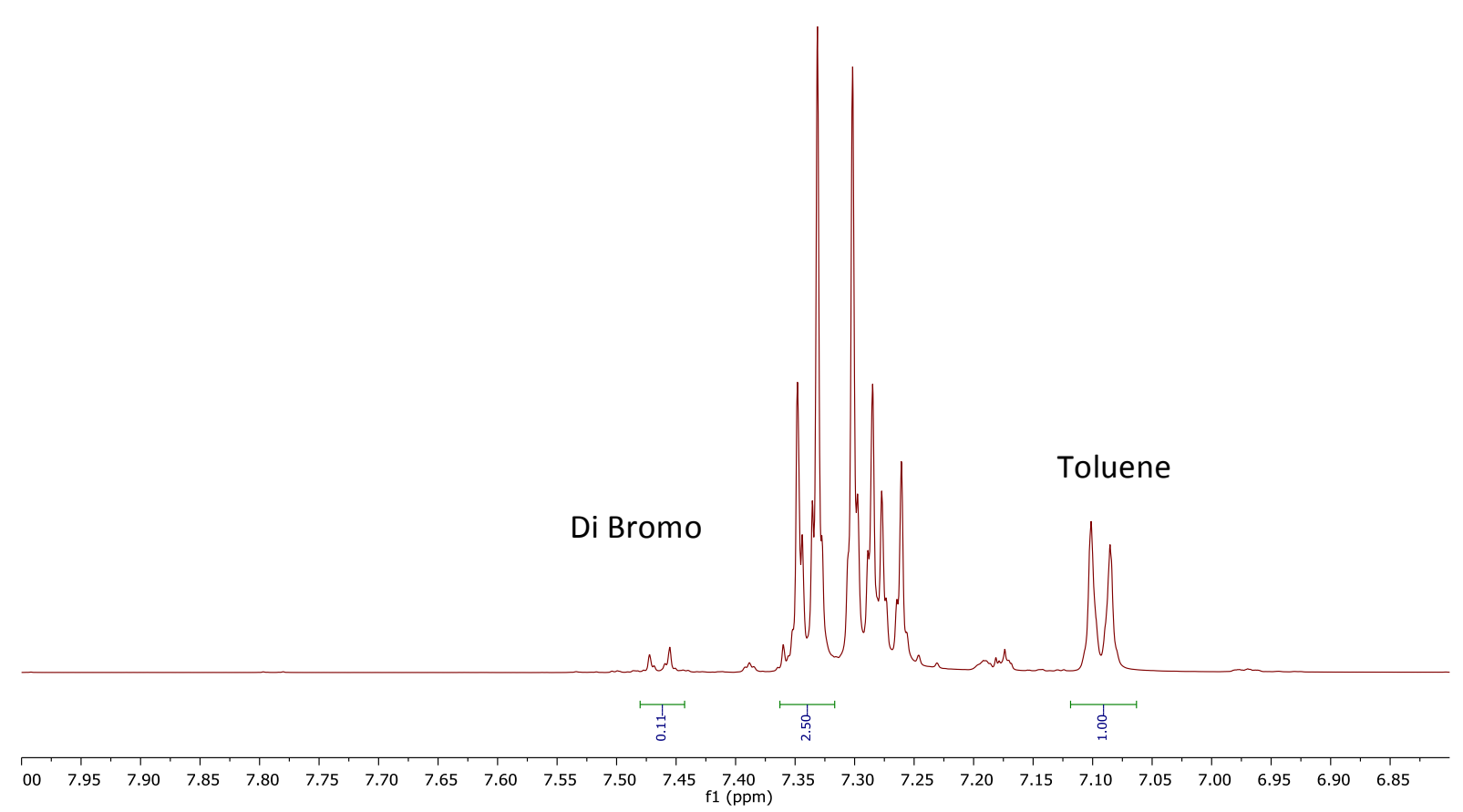

Conversion $=72 \%$, selectivity $=96 \%$ 
Crude NMR for conversion/selectivity for $2 \mathrm{c}$. Top, $0.5 \mathrm{M}$ reaction; bottom, $2 \mathrm{M}$ reaction

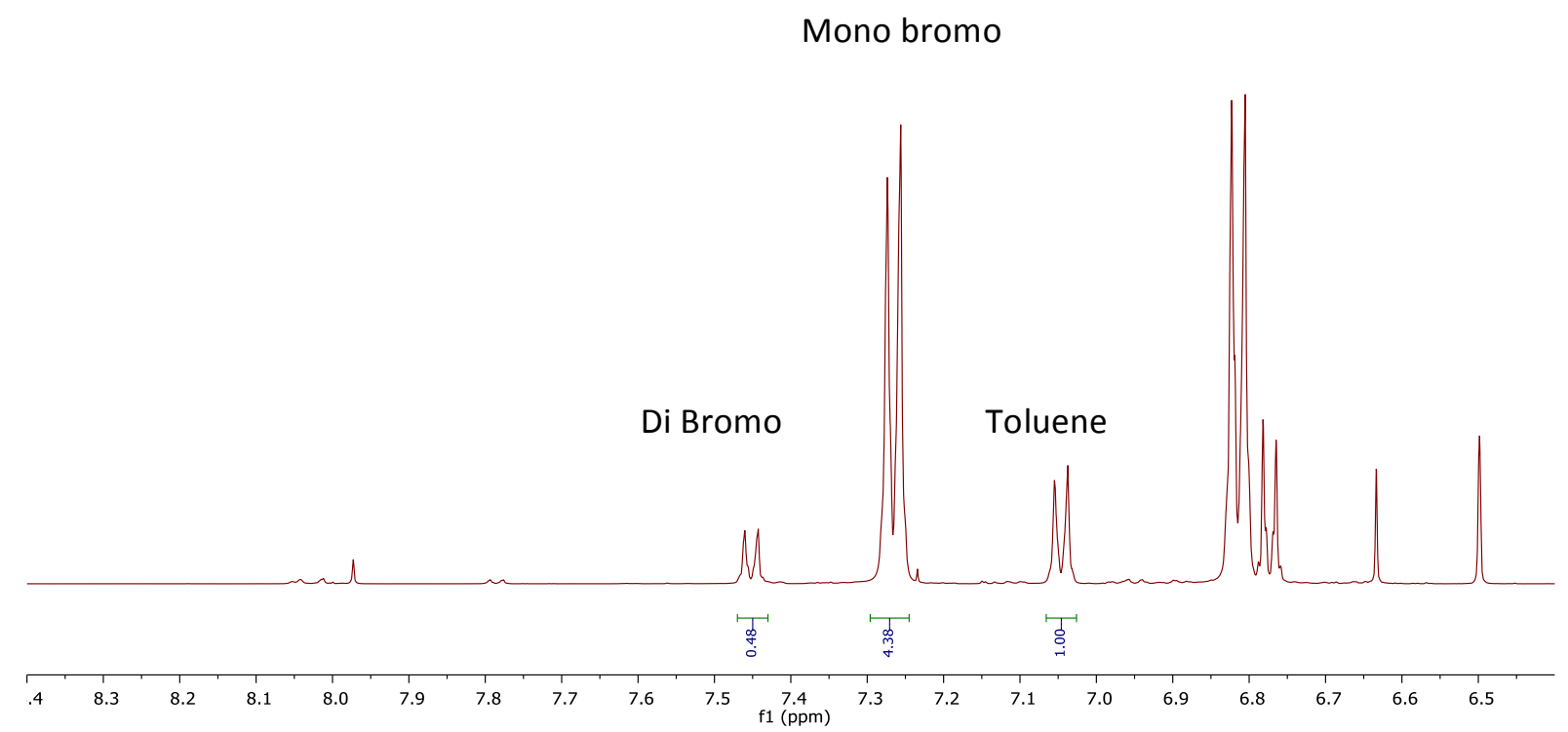

Conversion $=83 \%$, selectivity $=90 \%$

Mono bromo

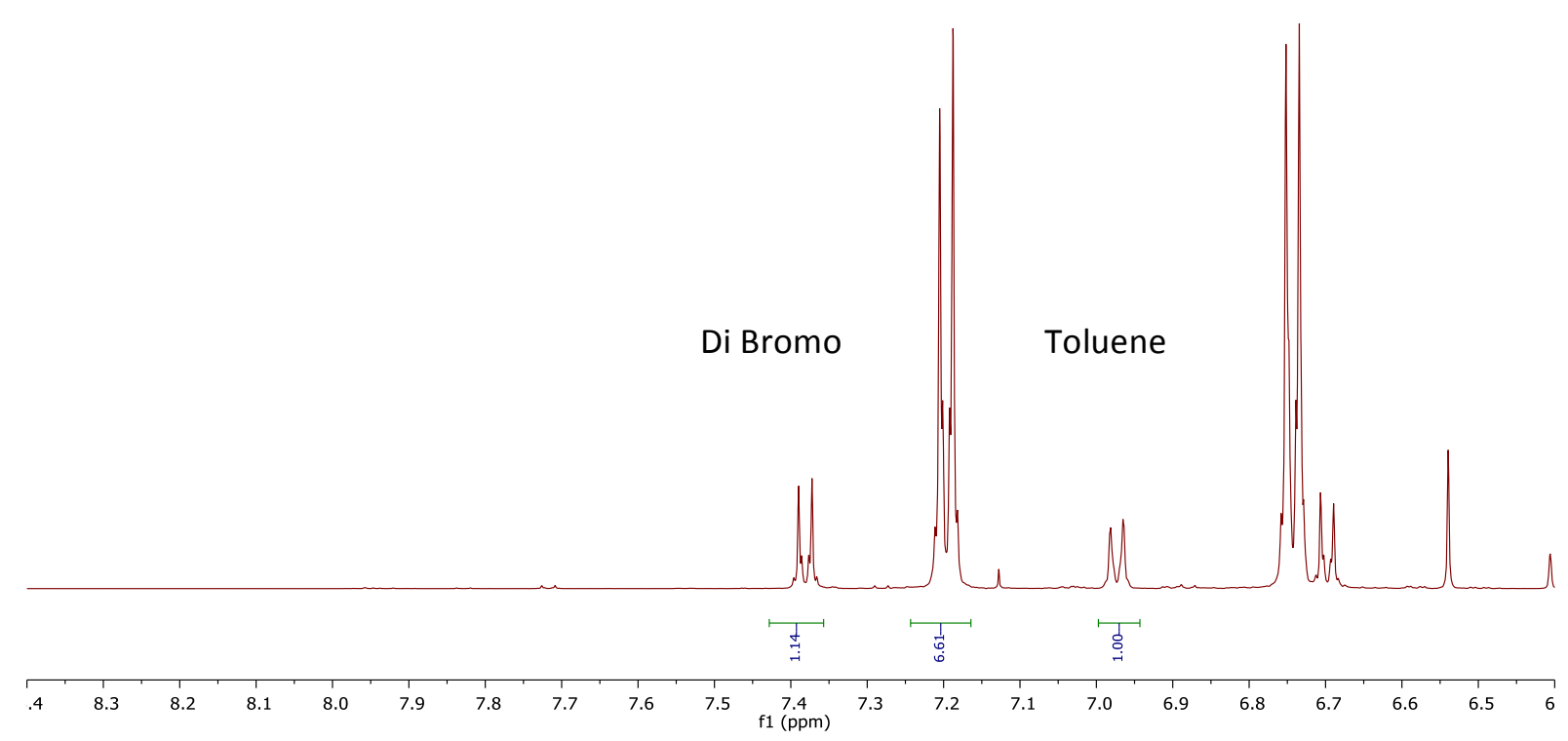

Conversion $=89 \%$, selectivity $=85 \%$ 
Crude NMR for conversion/selectivity for $2 \mathrm{~d}$. Top, $0.5 \mathrm{M}$ reaction; bottom, $1.5 \mathrm{M}$ reaction

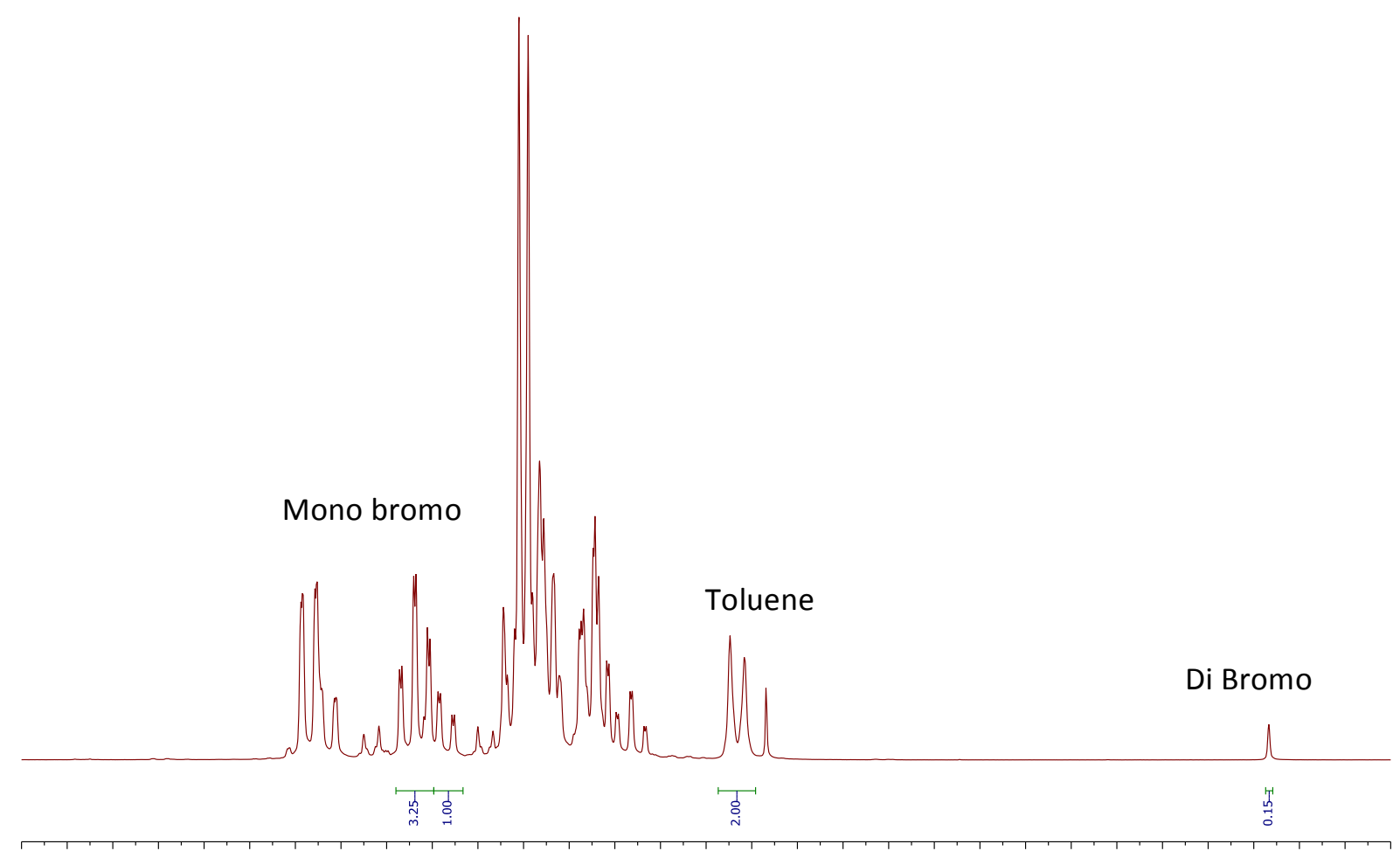

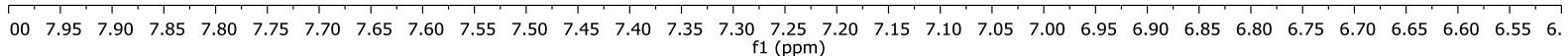
Conversion $=69 \%$, selectivity $=96 \%$

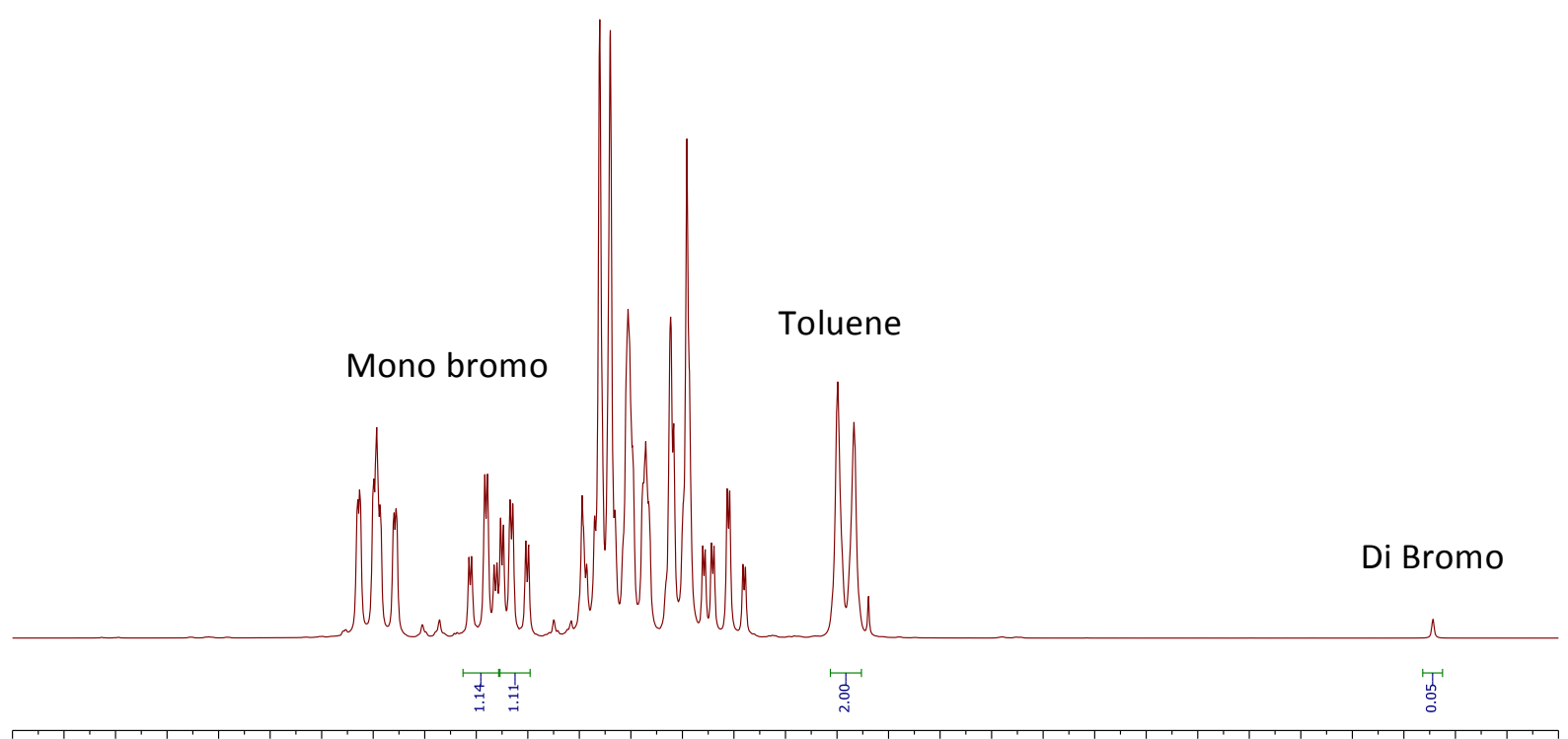

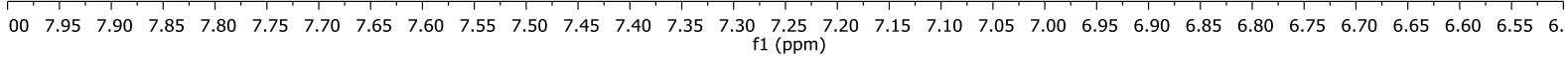
Conversion $=53 \%$, selectivity $=98 \%$ 


\section{References}

${ }^{1}$ Cantillo, D.; de Frutos, O.; Rincon, J. A.; Mateos, C.; Kappe, C. O. A Scalable Procedure for Light-Induced Benzylic Brominations in Continuous Flow. J. Org. Chem. 2014, 79, 223-229.

${ }^{2}$ Ni, S.; El Remaily, M. A. E. A. A. A.; Franzen, J. Carbocation Catalyzed Bromination of Alkyl Arenes, a Chemoselective $\mathrm{sp}^{3}$ vs. $\mathrm{sp}^{2}$ C-H Functionalization. Adv. Synth. Catal. 2018, 360, 4197-4204.

${ }^{3}$ Francis, D.; Nelson, A.; Marsden, S. P. Synthesis of $\beta$-Diamine Building Blocks by Photocatalytic Hydroamination of Enecarbamates with Amines, Ammonia and N-H Heterocycles. Chem. Eur. J. 2020, 26, 14861-14865. 